\title{
Plasma und Kernsubstanz in der normalen und der durch äußere Faktoren veränderten Entwicklung der Echiniden ${ }^{1}$.
}

Von

Emil Godlewski jun.

Aus der embryologischen Abteilung des anatomischen Instituts der Jagellonischen Unirersität in Krakau.

Mit Tafel III und IV und 2 Figuren im Text.

Eingegangen am 27. April 1908.

Inhaltsübersicht.

Seite

Einleitung. . . . . . . . . . . . . . . . . . 279

Untersuchungsmethoden . . . . . . . . . . . . . . . . . 281

I. Teil . . . . . . . . . . . . . . . . . . . . . . . . . 283

1. Die Zunahme der Kernsubstanz während der Entwicklung. . . . . 283

2. Der Einfluß der äußeren Faktoren auf die Kernsubstanzproduktion. 293

II. Teil. Der Verlauf der Entwicklung und der Kernsubstanzbildung bei der durch $\mathrm{CO}_{2}$-haltiges Seewasser gehemuten Plasmateilung. . . . . . . . 302

1. Methode and Untersuchungsaufgabe . . . . . . . . . . . . . . . 302

2. Die Veränderungen in den Befruchtungserscheinungen. . . . . . 303

3. Der Verlauf der Furchungsperiode, der Blastocoelbildung und Kernsubstanzproduktion . . . . . . . . . . . . 307

4. Die Mitosen der Riesenkerne (Syncaryonten' . . . . . . . . . . . 320

5. Andre Regulationstypen, weitere Entwicklung . . . . . . . . . . 322

6. Zusammenfassung der letzten Kapitel. . . . . . . . . . . . 323

Literaturverzeichnis . . . . . . . . . . . . . . . . . . . . . 324

Erkliirung der Abbildungen . . . . . . . . . . . . . . . . 326

Nachtrag . . . . . . . . . . . . . . . . 327

1 Torgetragen in der Sitzung der Akademie der Wissenschaften in Krakau am 9. Juli 1907. Vgl. Vorliufige Mitteilung Bull. d. IAcad. des Sc. de Cracovie Juin 1908. 


\section{Einleitung.}

Vor zwei Jabren habe ich in der Arbeit tiber die Bastardierung der Echiniden mit Crinoiden darauf bingewiesen, daß die Kreuzung zwischen diesen zwei Tierklassen Bastarde von rein mütterlichem Typus ergab und daß die arrhenocaryotischen Bastarde sich bis zum Gastrulastadium entwickelten und ebenfalls rein mittterliche Charaktere zur Schan trugen. Ohne die Rolle des Kerns bei den Vererbungserscheinungen in Abrede zu stellen, habe ich jedoch aus meinen Versuchen den Schluß gezogen, daß sowohl der Kern als auch das Protoplasma an dem Mechanismus der Vererbungserscheinungen sich beteiligen kann 1 .

Die Resultate meiner oben erwähnten Arbeit, welche auf die Wichtigkeit der Wechselbeziehung zwischen dem Kern und Protoplasma für das Vererbungsproblem hinzaweisen scheinen, und die, meiner Ansicht nach, sehr wichtige Arbeit von LoEB (05) haben mir die Anregung gegeben, das Verhältnis zwisehen dem Kern und Protoplasma während der Entwicklung näher zu untersuchen.

In den Angaben der bisherigen Literatur, besonders aber in denjenigen von R. Hertwig $(03,03)$, J. Loeb $(05,06,07)$ und T. Boveri $(05,07)$ wurde das wichtige Problem der Kernplasmarelation hervorgehoben. In bezug auf die Entwicklung ist es von prinzipieller Bedeatung, daß im Laufe der Entwicklung das Verhältnis zwischen der Quantität von Plasma und Kernsubstanz sich verändert, daß jedoch von einem gewissen Entwicklungsstadium ab dieses Verhältnis konstant bleibt. Es ist selbstverständlich, daß bei verschiedenen Tierspecies dieses konstante Verbältnis zwischen Quantität der Kern- und Plasmamasse sich anders gestaltet und nur bei denselben Tierspecies und in den analogen Zellarten konstant bleibt.

Interessant sind die Resultate der Vergleichung des Verhältnisses zwischen der gesamten Kern- und Plasmamasse in verschiedenen Entwicklungsstadien desselben Organismus. Bestimmen wir z. B. dieses Verhältnis im befruchteten, aber noch nngeteilten Echinus-Ei, sodann in den Furchungsstadien, in den Elementen des Blastula-, Gastrulaund Pluteusstadiums, so ersehen wir, daß das Quantum der Kern-

1) In der Literatur der zwei letzten Jahre wurde den Ergebnissen meiner Arbeit von verschiedenen Seiten auch andre Deutung gegeben. Ich hoffe in der nächsten Zeit Gelegenheit zu haben, auf dieses Thema wieder einzngehen und dabei auch die Einwïnde, von welchen einige mir unberechtigt erseheinen, näher zu prïfen. 
substanz im Laufe der Entwicklung zugenommen hat. Beriicksichtigt man aber hierbei, daß während dieser ersten Entwicklungsstadien keine Nahrung von außen von dem sich entwickelnden Organismus aufgenommen wird, daß im Gegenteil noch eine gewisse Quantität der lebenden Substanz für den Betrieb der Lebensprozesse rerbraucht wird, so gelangen wir zu dem Schluß, daß die Zunahme der Kernsubstanz auf Kosten der plasmatischen Substanz zustande kommt.

Dabei findet also derjenige ProzeB statt, welcher von LoEB (05) als Transformation des Protoplasmas in Kernsubstanz aufgefaßt wird. In Anbetracht dessen, daß die chemisehe Zusammensetzung des lebenden Protoplasmas und der lebenden Kernsubstanz ungenügend bekannt ist, und daß ans diesem Grunde auch der chemische Unterschied zwischen diesen beiden Substanzen sich nicht definieren läßt, ist es unmöglich, sich über die chemischen Reaktionen, welche das Wesen dieser Transformationsprozesse ausmacheu, eine bestimmte Ansicht zu bilden.

Auf Grund seiner Untersuchungen iiber die künstliche Parthenogenese ist LoEB $(06,07)$ zu dem SchluB gekommen, daB während der Furchung des Eies ein Oxydatiousprozeß stattfindet, welcher szweifellos in naher Beziehung zu der Synthese des Kernmaterials aus den Bestandteilen des Protoplasmas " steht.

LonB nimmt weiter an, daß die Anregung zur Entwicklung durch jene Prozesse bedingt ist, welche die genannten Oxydationsvorgänge auslösen und auf diese Weise die Synthese von Kernsubstanz ans der Plasmamasse veranlassen.

Abgesehen davon, ob die Synthese des Kernmaterials aus den Bestandteilen des Protoplasmas das Wesen der Entwicklungsvorgünge bildet, oder ob sie nur als Erscheinung aufzufassen ist, welche die Entwicklung beständig begleitet, mul ihr vom allgemein biologischen Standpunkte aus eine prinzipielle Bedeutung zugeschrieben werden. Und besonders heutzutage, wo die cytologischen Untersuchungen neben den experimentellen Forschungen in Vererbungsstudien das Hauptinteresse auf sich ziehen, wo das Problem der Lokalisation der *vererbungstragenden "Substanzen so viel diskutiert wird, scheint das Problem der Kerusubstanzbildung bei der Entwicklung sebr wichtig zu sein.

Die Ergebnisse der Untersuchungen von R. HeR'Twig (03), T. Boveri $\{05,06)$, Driesch $(98$, Marcus $\{06)$ u. a. haben sehr wichtige diesbeziigliche Tatsachen zutage gefördert. Die genaunten Autoren berücksichtigten jedoch hauptsächlich die Kernplasmarelation in ein- 
zelnen Zellen. Es schien mir wünschenswert, die Transformation des Protoplasmas in Kernsubstanz nicht nur in den einzelnen Zellen zu prüfen, sondern auch zu versuchen, die Gesamtsumme dieser in einzelnen Zellen verlaufenden Prozesse im ganzen embryonalen Organismus zu bestimmen.

Nicht ohne Bedeutung schien es mir auch, unter Berïcksichtigung der Anzahl der Bestandelemente des embryonalen Organismus, das Verhältnis zwischen der gesamten Kern- und Plasmasubstanzmenge in einzelnen Stadien der Entwicklung festzustellen.

Dabei miißte noch entschieden werden, ob die Faktoren, welche den ganzen Prozeß der Transformation des Plasmas in Kernsubstanz regulieren, der lebenden, sich entwickelnden Substanz selbst inhärent, bzw. durch ihre Struktur bedingt sind, oder ob die Transformationsprozesse auch von äußeren Faktoren beeinflußt werden können.

Um alle diese Fragen entscheiden zu können, bedarf es zahlreicher morphologischer und experimenteller Untersuchnngen. Die vorliegeude Arbeit bringt nur einen Teil des Tatsachenmaterials, welches rielleicht zur Lösung einiger Fragen auf diesem Gebiete beitragen kann.

Meine Arbeit wurde in Triest in der zoologischen Station begomnen und der größte Teil derselben in Neapel im Jahre 1907 ausgetiihrt.

\section{Untersuchungsmethoden.}

Als Untersuchungsmaterial dienten mir hauptsächlich die Eier von Echinus mirrotuberculatus; ein Teil der Experimente wurde auch an den Eiern von Strongylocentrotus lividus ausgeführt.

Die Untersuchung sollte in dem Vergleich der Kerngrößenveränderungen während verschiedener Entwicklungsstadien bestehen. Für jeden, welcher das Untersuchungsmaterial kennt, bedarf es keiner langen Motivierung, daß sich solche Untersuchungen am lebenden Material nicht durchführen lassen. Das Protoplasma, sogar das des Echimes-Eies, ist fur diese Untersuchung zu undurchsichtig, die Kerne, welche im unbefruchteten $\mathrm{Ei}$ sehr deutlich sichtbar sind, zeigen während der Entwicklung zu wenig scharfe Umrisse und verbleiben, besouders in den ersten Entwicklungsphasen, zu kurz im Ruhestadium, als daß man am lebenden Material den Umfang der Kerne genau bestimmen könnte. Am abgetöteten Material wird das Protoplasma noch undurchsichtiger, so daß ich nach verschiedenen Proben mich 
doch entschließen mußte, am fixierten Material za arbeiten. Ich bin mir vollkommen bewußt, daß die Ergebnisse solcher Bestimmungen keinen Anspruch auf besondere, absolute Genauigkeit haben können.

Doch in Anbetracht dessen, daß auch alle sonstigen bisherigen Ergebnisse auf dem Gebiete der Kernplasmarelation anf Grund ebensoleher, nur relativ genauer Beobachtungen gewonnen worden sind und daß es sich tatsächlich nar um relative Größen handelt, glaube ich durchaus berechtigt zu sein, aus meinen derartigen Untersuchungsresultaten gewisse Schltisse ziehen zu dürfen. Das Material wurde während verschiedener Entwicklungsstadien fixiert. Als Fixierungsmittel warde nur teilweise die Perenrische Flissigkeit ${ }^{1}$ ), meistens aber die Boverrsche Pikrin-Essigsäuremischung verwandt. Die Eier wurden nachher in den Glasröhrchen durch Alkohole von zunehmender Konzentration bis zum Alcohol absolutus durchgefuhrt, sodann in Celloidin uibertragen and schließlich in Paraffin eingebettet. Diese Methode ${ }^{2}$ ) ergibt sehr gute Resultate; der Vergleich mit Eiern, welche nur in Paraffin eingebettet waren, ergibt, daß bei der doppelten Einbettung die Schrumpfung anvergleichlich geringer ist. Die Präparate wurden auf den Objektträgern mittels des Heidexhatxschen Eisenhämatoxylin-Verfahrens gefärbt. Das Protoplasma wurde mit Bordeaux $R$ nachgefärbt.

Außerdem wurden Präparate verschiedener Entwicklungsstadien in toto hergestellt. Die Embryonen wurden mit Boraxkarmin gefärbt. Diese von BOvERI gewöhnlich angewandte Methode eignet sich am besten zur Bestimmung der Größe der Kerne in späteren Stadien.

1) Prof. Paul Mayer hat im Jahre 1898 (Grundziige der mikroskopisehen Technik. II. Auf. S. 34 Anm.) darauf hingewiesen, daß die Pereny ische Fliissigkeit eine ganz unrationell zusammengestellte Mischung ist. Die Chromsüure kann nämlich in dieser Mischung bei der Fixierung gar keine Rolle spielen, da sie sich schon vorher zersetzt. Auf Veranlassung des Herrn Prof. P. MaY'er habe ich einige diesbezügliche Versuche angestellt: Dasselbe Material wurde zu einem Teil in der Perexrischen, zum andern Teil in einer Mischung, welche bis auf die Chromsiure auf dieselbe Weise wie die Perenyische Flüssigkeit zusammengesetzt war, fixiert. Die Chromsüure wurde durch dasselbe Quantum destillierten Wassers ersetzt. Die Untersuchung des Materials hat vollkommen die Angaben von MaYer bestätigt, indem sowohl der eine wie der andre Teil des Materials vollkommen denselben Erhaltungszustand darbot.

2) Die Methode der doppelten Einbettung in Celloidin und Paraffin wurde von Herrn Biafaszewicz speziell für die Einbettung von Eiern näher ansgearbeitet, woriiber an andern Orten näher berichtet wird. 


\section{Teil.}

1. Die Zunahme der Kernsubstanz während der Entwicklung.

In der Einleitung habe ich schon darauf hingewiesen, daß von RrChard Hertwig der Begriff der „Kernplasmarelation «, d. i. Massenverhältnis von Protoplasma und Kernsubstanz, in die Literatur eingeführt wurde. Da es voraussichtlich viele Arten von Relation zwischen Kern und Plasma gibt, so wollen wir diese Relation genauer als die "quantitative Kernplasmarelation \&ezeichnen. Dieses Problem wurde von mehreren Autoren - Boveri (05, 07), Driesch $(98,06)$, Marcus (06), Gerassimow (02) - erörtert. Loeb hat in einer Reihe von äußerst wichtigen Publikationen auf die Bedentung der schon früher bekannten (BovERI, 92, S. 468) Tatsache hingewiesen, daß nicht nur das Verhältnis zwischen der Kern- und Protoplasmamenge in einzelnen Zellen während der Entwicklung eine Veränderung erfährt, sondern daß auch die absolute Kernsubstanzmenge im ganzen Organismus wïhrend des Entwicklungsverlaufes zunimmt.

Angaben in bezug auf die Quantität der Kernsubstanz in ihrer absoluten ursprünglichen Menge, wie auch in bezug auf die Veränderungen der Kernsubstanzquantität in den Stadien ihrer Weiterbildung fehlen - meines Wissens - überhaupt in der einschlägigen Literatur.

Als Ausgangspunkt meiner Untersuchungen diente mir das unbefruchtete reife Ei von Echinus. Die Umrisse des Eies wurden nach dem gefärbten Präparat mit der Camera entworfen (Zeiss $2 \mathrm{~mm}$ hom. Immersion, Komp.-Oc. 6, Tubuslänge 155, die Höhe des Objekttisches, was genau der 1000 fachen Vergrößerung entspricht); auf der Zeichnung (rgl. Textfig. 1) wurde der Durchmesser, bzw. zwei Durchmesser, nach dem Millimetermaßstab gemessen ${ }^{1)}$ und daraus das Volumen des Eies bzw. des Kerns berechnet. Die Eier haben größtenteils ellipsoidische oder kugelige Gestalt. Im letzteren Fall gentigt die Bestimmung des Durchmessers und das Volumen kann nach der Formel $v=4 / 3 \pi r^{3}$ ausgerechnet werden. Wenn das Ei jedoch die Form eines Ellipsoids zeigte, habe ich zwei Durchmesser bestimmt; da der dritte nicht direkt bestimmt werden konnte, wurde er als dem kleineren Durchmesser

1: Alle Ziffern sind also in Millimetern angegeben und beziehen sich nicht auf das Objekt selbst, sondern auf die unter der oben angegebenen Vergrößerung entworfene Zeichnung des betreffenden Objektes. 
gleich angenommen. Das Volumen wurde in diesem Fall nach der Formel $v=\frac{\pi}{6} d_{1}^{2} d_{2}$ ausgerechnet, wo $d_{1}$ den kleineren, $d_{2}$ den größeren Durchmesser bezeichnet.

Der längere Durchmesser des ganzen Eies - Nittelwert aus zehn Messungsresultaten (95, 95, 98, 90, 98, 88, 94, 93, 90, 92) - beträgt 93,3, der kleinere Durchmesser $(8 \check{0}, 92,90,88,92,82,85,83,82,92$;

Textfig. 1.

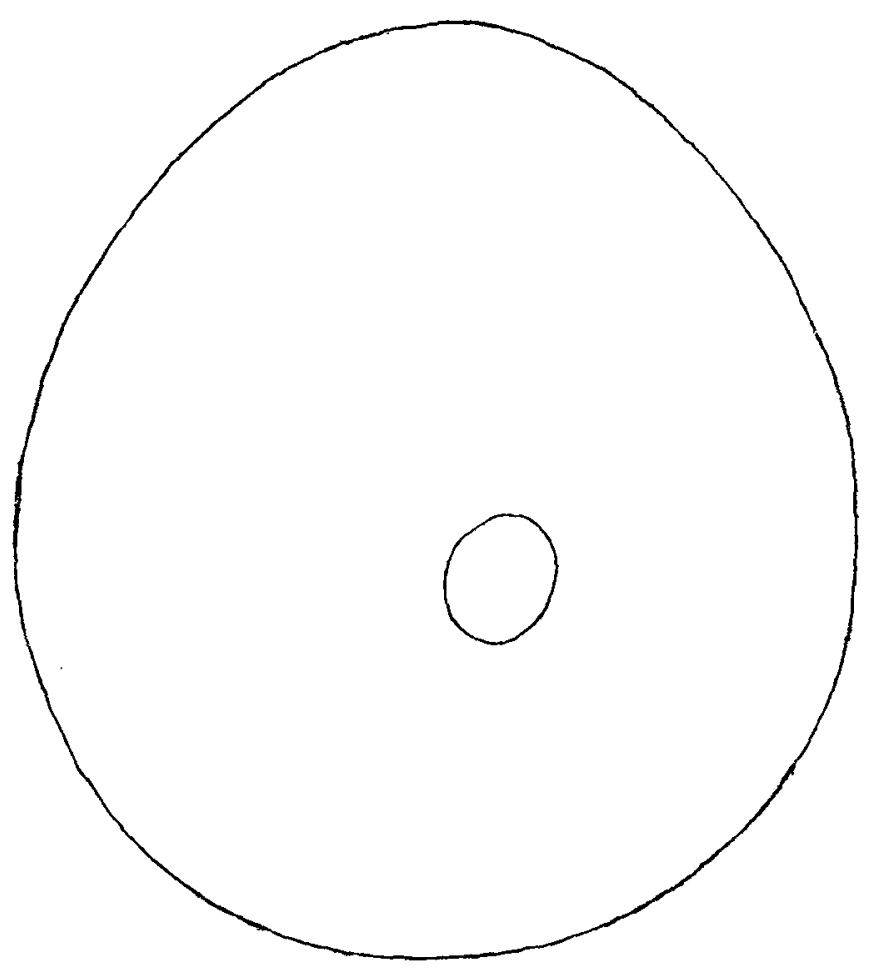

beträgt im Mittelwert 87,1. Der längere Durchmesser der Kerue (11, $13,14,11,11,11,11,11,12,12)=11,7$, der kleinere $(11,11,11,11$, $9,8,9,11,10,12)=10,3$ (vgl. Fig. 1). Das Eivolumen beträgt also 368770, das Kernvolumen 650. Das Verhältnis zwischen Protoplasma und Kernsubstanz ist also wie $567: 11$ ), abgerundet $550: 1$. Nach

1) Dieses Verhältnis habe ich auch am lebenden Material bestimmt. Es hat sich dabei herausgestellt, daß es durch die Fixielungsmittel nicht sebr stark veründert ist. Nach den Bestimmungen am lebenden Naterial beträgt es ó13:1. 
der Befruchtung verdoppelt sich bekanntlich die Chromatinmasse. Das Chromatin, welches in dem Spermatozoonkopf in das Ei eingeführt wurde, ist jedoch bei den Echiniden bekanntlich stark kondensiert. Sogar nach der Verschmelzung der Vorkerne bleibt das väterliche Chromatin im Copulationskern bis zum Verschwinden der Kernmembran kondensiert, so daß eine Zunahme des Kernvolumens nach der Copulation durch Messungen sich nicht feststellen läßt. Nur in den Fällen, in welchen das Vorriicken des männlichen Vorkerns künstlich verlangsamt wird und infolgedessen der männliche Vorkern bedeutender aufquillt (Fig. 9, welche aber unter andrer Vergrößerung gezeichnet wurde; kann nach Verschmelzung der Vorkerne ein Unterschied zwischen dem Volumen des weiblichen Vorkerns und dem Volumen des Copulationskerns festgestellt werden.

Daß eine Verdoppelung der Kernsubstanzmenge während des Befruchtungsprozesses eintritt, läßt sich ohne weiteres an den Eiern jener Tiere (Asteriden, Physa, Ascaris usw.) feststellen, bei denen aach der Befruchtung der männliche Vorkern während seiner Wanderung durch das Eiprotoplasma regelmäßig zu der gleichen Größe wie der Eikern zunimmt. Diese Tatsache bedarf also keiner längeren Exörterung. Die Verdoppelung der Kernsubstanzmenge während des Befruchtungsvorganges ist jedoch nicht ausschließlich anf die Kernsubstanz zuriuckzuführeu, welche im Spermakern in das Ei eingefuhrt wurde; ein Teil derselben, nämlich der Kernsaft des männlichen Vorkerns, stammt aus dem Eiprotoplasma; nur das Chromatin des Furchungskerns stammt zu gleichen Teilen aus beiden Vorkernes.

Das quantitative Verhältnis zwischen der Plasma- und der Kernsubstanzmenge wird also im befruchteten Ei ungefähr $550: 2$ betragen.

Es folgt die erste Mitose. Im Zweizellenstadium habe ich die Blastomerenkerne gemessen. Der Mittelwert (aus zehn Bestimmungen, vgl. Fig. 2) des größeren Durchmessers beträgt 13, des kleineren 10,1. Das Kernvolumen beträgt also 691. Daraus sehen wir, daß das Volumen jedes Blastomerenkerns im Zweizellenstadium dem Volumen des weiblichen Vorkerns (650) sehr nahe steht.

Wenn wir unter der Transformation des Protoplasmas zur Kernsubstanz die Bildung der gesamten Kernbestandteile verstehen, so mulB daraus der Schluß gezogen werden, daß der ersten Kernteilung die Transformation der plasmatischen Substanz zu Kernsubstanz nicht rorangeht, da der Zuwachs der chromatischen Substanz auf das Eindringen des Spermakopfes in das Ei zurïckgeführt werden muß und die Bestandteile des Protoplasmas nur an der Zunahme der Kern- 
saftmenge teilgenommen haben. Das Verhältnis zwischen der gesamten Plasmamenge und der gesamten Kernsubstanzquantität im ganzen Embryo bleibt auf dem $Z_{\text {weizellenstadium dasselbe }}$ wie im befruchteten Ei, also wie 550:2. Daraus geht hervor, daß dieses Kernsubstanzmaterial, welches im befruchteten Ei vorhanden war, ohne irgend eine Zunahme während der ersten Furchungsphase in zwei Hälften geteilt wurde.

Die im nächsten Entwicklungsstadium, d. h. im Vierblastomerenstadium, unternommenen Messungen ergaben keinen durchgreifenden Unterschied zwischen der Größe der einzelnen Blastomerenkerne und der Größe des weiblichen Vorkerns. Ein Blick auf die Fig. 3 genügt, um sich zu tiberzeugen, daß die Dimensionen der Kerne nicht sehr stark verändert worden sind. Der Mittelwert aus zehn Bestimmungen der Kerndimensionen (vgl. Fig. 3, 4) beträgt für den längeren Durchmesser 11,5, für den kleineren 9,3. Das Volumen des Kerns im Vierzellenstadium beträgt also 521 . Infolgedessen hat sich selbstverständlich das Verhältnis zwischen der gesamten Plasma- und Kernsubstanzmenge zugunsten der letzteren verschoben. Die gesamte Kernsubstanzmenge beträgt im Vierzellenstadium $4 \times 521=2084$. Die Zunahme der Kernsubstanzmenge ist offenbar das Resultat des Transformationsprozesses des Protoplasmas zu Kernsubstanz; diese Erscheinung läßt sich also im Laufe der Entwicklung zum erstenmal während der Bildung der Kerne im Vierzellenstadium feststellen.

Dieser Transformationsprozeß schreitet während der nächsten Furchungsphasen mit noch zunehmender Intensität fort, da er durch immer größere Kernenanzahl geleitet wird. Die Bestimmung der Kerndimensionen im 32-Zellenstadium ergab den Mittelwert aus zehn Messungen (vgl. die Fig. 4, welche die Kerngrößen darstellt) des längeren Durchmessers 11,9, des kleineren 10,0. Das Kernvolumen beträgt also 623. Wir sehen also, daß noch auf diesem Furchungsstadium die Kerngröße sich von dem Volumen des weiblichen Vorkerns (650) beinahe nicht unterscheidet.

Die Größe der Kerne des 64-Zellenstadiums stellt die Fig. 5 dar. Das Volumen eines Kerns dieses Stadiums beträgt 473, man sieht also hier die beginnende Verkleinerung der Kerne, welche mit fortschreitender Furchung immer deutlicher hervortritt.

Das Kernvolumen in Blastulastadium in der Zimmertemperatur und im gewöhnlichen Seewasser beträgt im Durchschnitt nach meinen Berechnungen 24,45. Um die absolute Kernsubstanzmenge zu berechnen, mußte vorher die Anzahl der Zellen festgestellt werden, aus 
denen die Wand der Blastula zusammengesetzt ist. Zu diesem Zweck wurde die Kernanzahl im größten Querschnitt der Blastulawand bestimmt. Diese Zahl schwankt zwischen $60-67$. Auf der Textfigur 2 sehen wir 67 Kerne. Durchschnittlich beträgt also diese

Textfig. 2.

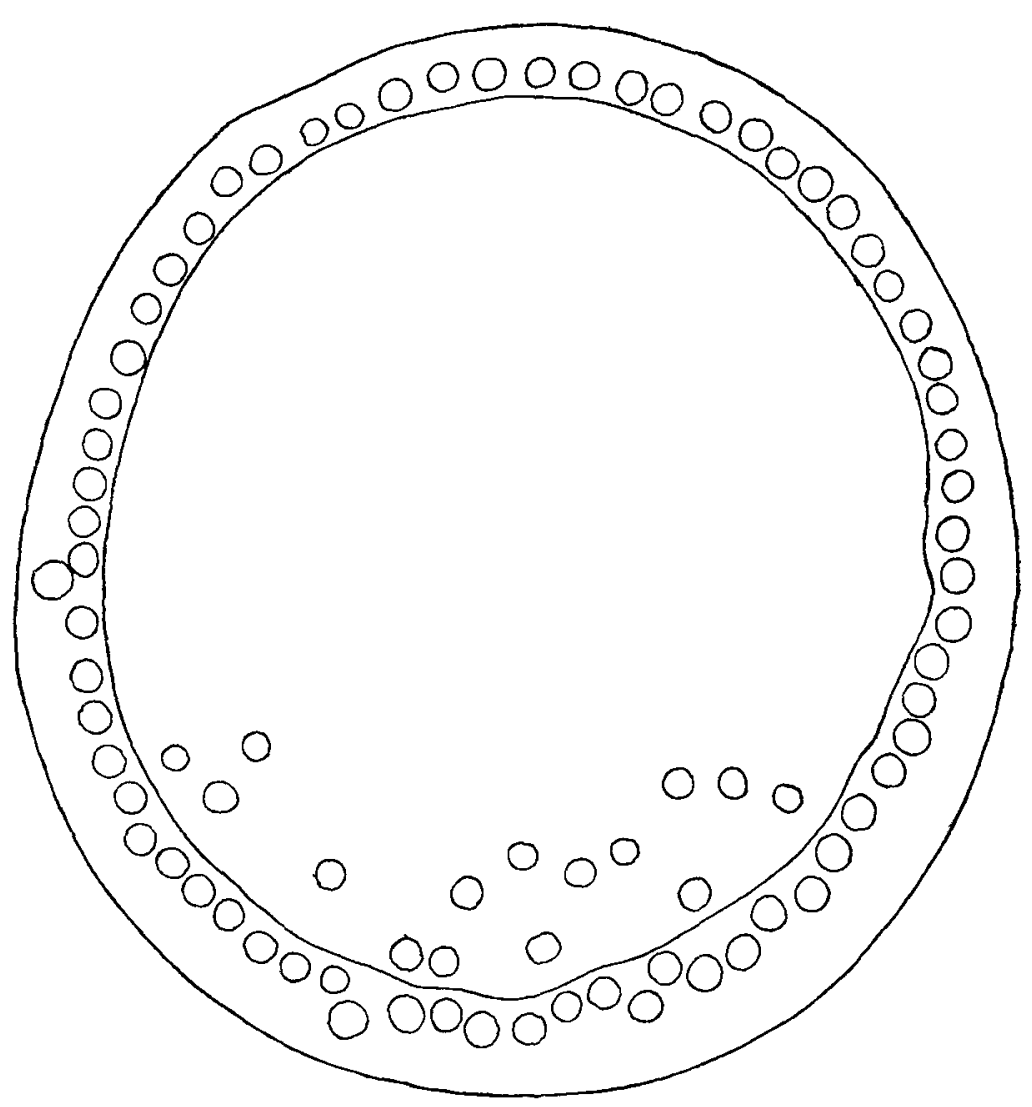

Zahl 63 Kerne. In bezug darauf läßt sich der Radius nach der Formel:

$$
T=63=2 \pi r
$$

ausrechnen :

$$
r=10 \text {. }
$$

Daraus läßt sich wieder die Anzahl der Kerne, welche auf der Oberfläche der Kugel bzw. in der Blastulawand liegen, nach der Formel berechnen: $0=4 \pi r^{2}=1256$. 
Die Zahl der Kerne beträgt also in der Blastulawand $1256^{1}$ ) und die absolute Kernsubstanzmenge $1256 \times 24.45=30716$.

Für die von uns erörterten Fragen ist noch das Verhältnis zwischen der gesamten Plasmamenge zu der absoluten Kernsubstanzmenge von Wichtigkeit.

Die absolnte Plasmamenge, aus welcher die Blastulawand besteht, läßt sich berechnen, wenn man die Blastula als Hohlkugel betrachtet. Aus dem Volumen, welches auf Grund des Durchmessers des äußeren Blastulaumfanges berechnet werden kann, und aus dem Durchmesser des Blastocoels kann das Volumen der Blastulawand festgestellt werden. Zieht man das Volumen der gesamten Kerne ab, so ergibt sich die absolute Plasmamenge der Blastulawand. Sie beträgt nach meinen Präparaten 201730. Das Verhältnis zwischen der absoluten Plasmamenge und der absoluten Kermsubstanzquantität beträgt also $201730: 30716$ oder $6: 1$.

Aus dem Vergleich der Zunahme der Kernsubstanz in den successiven Furchungsstadien ergibt sich, daß sie während der ersteu Entwicklungsstadien sehr stark, ungeführ in geometrischer Progression, an Quantität zunimmt, wobei sich entsprechend auch das Verbältnis zwischen der gesamten Plasma- und Kernsubstanzmasse zugunsten der letzteren verschiebt. In der S. 289 angegebenen Tabelle sind die Ziffern angeführt, um zu zeigen, wie die Kernsubstanz an Quantität zunimmt und wie sie zunehmen sollte, wenn der Zuwachs derselben in successiven Furchungsstadien genal in geometrischer Progression vor sich gehen würde. Endlich ist in der Tabelle auch die Veränderung des Verhältnisses zwischen der gesamten Plasmamasse und der absoluten Kernsubstanzmenge angegeben.

Der ganze FurchungsprozeB kann, wie aus der Tabelle zu ersehen ist, in zwei Hauptphasen geteilt werden. In der ersten Furchungsphase, welche sich bei Erhinus vom Zweizellenstadium bis zum 64-Zellenstadium erstreckt, wird die Kern-

1: Bekanntlich hat Driesch ,00, anf Grund seiner Untersuchungen iiber die isolierten Blastomeren die Rechnungen durchgeführt. nach welchen das ideale Blastulastadinm ans 1024 Zellen bestehen würde. Die direkte Beobachtung ergab aber, daß die vier Micromeren nur 40 Abkümmlinge liefern; deswegen tritt nach Driesch die Blastulation in einem Stadium ein, in welchem 808 Zellen vorhauden sind. Die Differenz zwischen meinen Ergebnissen und denjenigen von Driesch hat vielleicht in den :inßeren Bedingungen und individuellen Schwankungen (vgl. PETER, 05, im Volumen der Eier ihren Grund, oder. was sehr leicht müglich ist, darin, daß von mir and DRIEsch nicht genan gleiche Stadien in Betracht gezogen worden sind. 
Tabelle I.

\begin{tabular}{|c|c|c|c|}
\hline $\begin{array}{c}\text { Bezwehmung } \\
\text { Furs } \\
\text { Furchungstadium }\end{array}$ & $\begin{array}{l}\text { Das vorgefundene } \\
\text { Volumen } \\
\text { der gesamten } \\
\text { Kernsubstanzmass }\end{array}$ & $\begin{array}{l}\text { Das Volumen der gesamten } \\
\text { Kernsubstanz berechnet auf Grund } \\
\text { der Voraussetzung, dab die Kern- } \\
\text { masse in den successiven Fur- } \\
\text { chungsstadien genau naeh der } \\
\text { geometrischen Progression zu- } \\
\text { nimmt }\end{array}$ & $\begin{array}{l}\text { Das Verbältnis der } \\
\text { gesamten Plasma- } \\
\text { zu der gesamten } \\
\text { Kernsubstanzmenge }\end{array}$ \\
\hline Unbefruchtetes $\mathbf{E i}$ & 650 & 650 & $550: 1$ \\
\hline Das befruchtete Ei & 1300 & 1300 & $27 \tilde{0}: 1$ \\
\hline Zweizellenstadium & 1382 & 1300 & $275: 1$ \\
\hline Vierzellenstadium & 2084 & 2600 & $177: 1$ \\
\hline 32-Zellenstadium & 19938 & 20800 & $18: 1$ \\
\hline 64-Zellenstadium & 30262 & 41600 & $12: 1$ \\
\hline Blastulastadium & 30716 & - & $6: 1$ \\
\hline
\end{tabular}

substanz produziert und ihre Quantität wächst von einem Stadium zum andern beinahe in geometrischer Progression, mit Ausnahme der letzten Zellgeneration (64 Zellen), in welcher dieser Zuwachs schon schwächerwird. Die zweite Periode der Furchung umfaßt die Furchungsstadien nach dem 64-Zellenstadium bis zur Blastulaausbildung.

In dieser Furchungsperiode findet die weitere Kernsubstanzproduktion fast nicht mehr statt und die Kernteilungen, welche in dieser Furchungsetappe verlaufen, fuhren also nur die Umarbeitung der Kernsubstanz durch. Die gesamte Kernsubstanz, welche in der ersten Furchungsperiode hauptsächlich durch die Transformation des Protoplasmas zu Kernsubstanz entstanden ist, war am Schluß dieser ersten Periode nur in verhältnismäßig wenigen Kernen gruppiert. Als Endpunkt der zweiten Furchungsperiode ist die Blastula aufufassen. Die Kernsubstanz ist in diesem Entwicklungsstadium auf etwa 1250 Kerne verteilt.

Was während der zahlreichen aufeinander folgenden Kernteilungen. der zweiten Furchungsperiode geschieht, läßt sich meiner Ansicht nach besser verstehen, wenn man die Bedeutung dieser Prozesse an der Hand unsrer Kenntnisse über die chromatische Substanz erwägt. Geht man nämlich bei den Betrachtungen von dem Standpunkte der Individualitätshypothese der Chromosomen von Boverr, oder von der Manörrierhypothese von Fıck, oder von irgend einer andern Auffassungsart aus, so stimmen doch die verschiedenen Auffassungen darin uberein, daß die Zahl der Chromosomen, welche während der 
mitotischen Vorgänge in verschiedenen Entwicklungsstadien derselben Tierspecies zum Vorschein kommen, konstant ist.

Nach der bisher allgemein anerkannten Anschauung ${ }^{1)}$ unterscheidet sich die Größe der Chromosomen während der Mitose in späteren Furchungsstadien nicht wesentlich von der Größe der Chromosomen der frühen Furchungscaryokinesen.

Wenn also die einzelnen Kerne der ersten Furchungsstadien ungefähr dieselbe Quantität der Chromatinsubstanz enthalten wie die Blastulazellenkerne, so geht daraus klar hervor, daß in der Kernsubstanzmasse der Blastulawand mehr Chromatin enthalten ist als iu derselben Kernsubstanzmenge des 64-Zellenstadiums.

Ich habe im Vorhergehenden den Nachweis geführt, daß während der ersten Furchungsperiode (bis 64 Zellen) fast die gesamte Kernsubstanzmenge, welche nachber auch am Ende der Furchung wahrnehmbar ist, sich schon ausgebildet hat. Wenn wir jetzt nach den letzten Erörterungen zu der Frage zurückkehren, was in den Teilungen der zweiten Furchungsperiode geschieht, so sind wir, meiner Ansicht nach, zu der Behauptung berechtigt, daß, obschon während der zweiten Furchungsperiode die Kernsubstanz nur wenig an Quantität zunimmt, sich doch ihre Zusammensetzung verändert, indem sie bei der Verteilung auf mehrere Kerne reicher an Chromatin wird. Dabei ist noch zu beachten, daß, wenn dieselbe Kernsubstanzmasse anf mebrere Kerne verteilt ist, die Oberfläche des Kernapparates bedeutend vergröBert und infolgedessen die wechselseitige Wirkung zwischen Kern und Plasmasubstanz erleichtert wird.

Die Veränderungen in dem Verhältnisse zwischen Plasma und Kernsabstanz während der Furchung bedürfen noch einer kurzen Besprechung. Dieses Problem kann ron doppeltem Standpunkte aus betrachtet werden: entweder mit Bezug auf das Verhältnis vom gesamten Plasma zur Kernsubstanzquantität oder auf die Relation ron Plasma und Chromatinmasse im ganzen embryonalen Organismus. Sowohl im ersten wie im zweiten Fall bildet das unbefruchtete Ei den Ausgangspunkt der Betrachtungen. Bestimmt man das, was R. HeRTwig Kernplasmarelation im unbefruchteten Ei nennt, so ist, wie oben angegeben wurde (vgl. S. 284), das Verhältnis zwischen der Plasma- und der Kernsubstanzquantität 550:1. Bestimmt man die Kernplasmarelation in den einzelnen Blastulazellen, so beträgt sie $6: 1$. Diese quantitativen Bestimmungen scheinen aus diesem Grunde

1: Vgl. Nachtrag zu dieser Arbeit. 
nicht ohne Bedeutung zu sein, weil neuerdings von einigen Autoren angegeben wurde, daß die Fixierung der Kernplasmarelation als die Furchung beendigendes Moment aufzufassen ist. Auf diese Frage werden wir noch später eingehen.

Die Verhältnisse zwischen der absoluten Plasma- und der gesamten Kernsubstanzmenge gestalten sich nach Beendigung der Furchung ebenfalls grundverschieden (550:1 im unbefruchteten Ei, $6: 1$ in Blastulastadium). Beruicksichtigen wir jedoch, was im Vorhergehenden tiber Zunahme von Kernsubstanz im allgemeinen und iiber Zunahme der chromatischen Substanz während der Furchung gesagt wurde, so kommt man zu dem Schluß, daß am Ende der ersten Furchungsperiode das Verhältnis zwischen der gesamten Plasma- und Kernsubstanzmasse schon der definitiven Fixierung nahe steht. Wir haben gehört, daß während dieser Periode fast die gesamte Kernsubstanz sich bereits ausgebildet hat und die Produktion derselben in der zweiten Furchungsetappe nur unbedentend fortschreitet. Wenn das Verbältnis zwischen der gesamten Plasma- und Kernsubstanzmenge in der zweiten Furchungsperiode sich noch weiter zugunsten der Kernsubstanz rerschiebt (im 64-Zellenstadium 12:1, im Blastulastadium $6: 1$ ), so geschieht es nicht wegen der weiteren Kernsubstanzproduktion, sondern wegen der Abnahme der Plasmamenge, was wahrscheinlich mit der Chromatinbildung im Zusammenhang steht.

Die Fixierung des Verhältnisses zwischen dem Plasma und der Chromatinsubstanzmasse fällt erst mit Beendigung des ganzen Furchungsprozesses zusammen. Aus dem Vorhergehenden ist bekannt, daß bei der Veränderung der Verteilnng der Kernsubstanz auf größere Kernenanzahl die Kernsubstanz an Chromatin reicher wird. Diese Zunahme des Chromatins in der Kernsubstanz schreitet bis zum Ende der Furchung fort, deswegen wird erst im Blastulastadium das Verhältnis zwischen der gesamten Plasma- und der gesamten Chromatinsubstanz normiert. Die Kernplasmarelation im HeRTwigschen Sinne verschiebt sich innerhalb einzelner Zellterritorien während der ganzen Furchung zugunsten der Kernsubstanz.

Bestimmt man die Kernplasmarelation in einzelnen Zellen der Blastulawand, so ergibt sich, daß das Verhältnis zwischen dem Plasma und Kern wie 5 bis $6: 1$ ist. Wir haben im Vorhergehenden gesehen, daß das Verhältnis der gesamten Plasmamasse zu der gesamten Kernsubstanzquantität sich wie $6: 1$ verhält. Die Übereinstimmung dieser beiden Verhältnisse ist also ziemlich genau.

Im Gastrulastadium und in den folgenden Entwicklungsphasen 
(Prisma, Pluteus) läßt sich die Plasmaquantität in Anbetracht der technischen Schwierigkeiten in den Messungen dieser geometrischen Formen nicht leicht durchführen. Infolgedessen ist auch die Frage nach dem Verhältnis zwischen Kern und Plasmamasse der direkten Untersuchung sehwer zugänglich ${ }^{1}$ ).

Was jedoch das Verhalten der Kernsubstanz anbelangt, so haben die Messungen der Kerndimensionen ergeben, daß die Kerne in diesen Entwicklungsphasen seit dem Blastulastadium nur wenig oder gar nicht an Volumen abgenommen haben.

Von Schmidt (04) und von Marccs (06) ist angegeben worden, daß die Kernvolumina der Plutei im Vergleich zu denjenigen der Blastulae kleiner sind. In vieien Fällen läßt sich eine geringe Verkleinerung der Kerne wirklich nachweisen. Ich habe jedoch sehr viele Kulturen beobachtet, in welchen dieser Unterschied entweder verschwindend klein war oder überhaupt sich nicht nachweisen ließ. Die unter derselben Vergrößertung entworfenen Skizzen beweisen, daf, abgesehen von den oft vorkommenden Abnormitäten, die Größe der Kerne im Pluteusstadium (Fig. 7) sich oft von derjenigen im Blastula stadium (Fig. 6) nicht wesentlich unterscheidet.

Fragen wir jetzt nach der absoluten Kernsubstanzmenge und der absoluten Chromatinquantität im Pluteusstadium, so können wir wenigstens indirekt den Beweis erbringen, daß diese Substanzen an Menge zugenommen haben. Obschon wir keine beträchtlichere Zunahme an Volumen in den einzelnen Kernen konstatieren können, so liegt doch in der Tatsache, daß während der Gastrulation und der Pluteusbildung die mitotischen Figuren im embryonalen Gewebe vorkommen, der Beweis dafür, daß die Zahl der Kerne wächst; und da sich die Kerne nicht bedeutend verkleinern, so muß die absolute Kernsubstanzmasse ebenfalls zunehmen.

Fassen wir jetzt unsre bisherigen Ergebnisse iiber das wechselseitige Verhältnis zwischen dem Protoplasma und der Kernsubstanz im embryonalen Organismus zusammen, so ergibt sich:

1) Die erste Kernteilung des befruchteten Eies bewirkt nur die Verteilung der in einer Zelle vorhandenen Kernsubstanz auf zwei Blastomeren. Die einzelnen Kerne des Zweizellenstadiums enthalten dieselbe Kernsubstanzquantität wie das unbefruchtete Ei.

2) Die rege Transformation der plasmatischen Substanz in Kernsubstanz kommt im Vierzellenstadium zum erstenmal in der Entwicklung zum Vorschein.

1) Genauere Bestimmung ließe sich vielleicht nur mit Hilfe der Modellierangsmethode durchfiihren. 
3) In der ersten Hälfte der Furchungsperiode (bis 64 Zellen) wird durch die Transformation des Protoplasmas in Kernsubstanz, welche in geometrischer Progression von Stadium zu Stadium zunimmt, fast die ganze Menge der Kernmasse, welche im Blastulastadium vorhanden ist, schon ausgebildet.

4) Während der Kernteilungen der zweiten Furchungsperiode (nach 64 Zellen) wird die in der ersten Furchungsperiode ausgebildete Kernsubstanz als Ganzes auf eine successiv von Stadium zu Stadium auwachsende Zahl von Kernen verteilt, wobei sich die Kernsubstanz an Chromatin bereichert.

5) Das Verhältnis zwischen der gesamten Plasma- und Kernsubstanzmasse wird am Ende der ersten Furchungsperiode der Norm genähert, das Verhältnis zwischen der gesamten Chromatin- und der gesamten Plasmamasse des ganzen embryonalen Organismus wird erst am Ende der Furchung im Blastulastadium fixiert.

6: Im Gastrula- und im Pluteusstadium nehmen die Kerne an Volumen nicht bedeutend $a b$ und da die Anzahl der Kerne zunimmt, so muß daraus der Zuwachs der gesamten Kernsubstanzmenge gefolgert werden.

2. Der Einfluß der äußeren Faktoren auf die Kernsubstanzproduktion.

Aus den bisherigen Erörterungen geht hervor, daß die embryonaleu Organismen in verschiedenen Entwicklungsstadien durch bestimmte Mengen der Kernsubstanz charakterisiert sind, daß sie jedoch innerhalb derselben in verschiedenen Entwicklungsstadien verschiedene Quantität an Chromatin enthalten. Wir haben weiter gesehen, daß die Quantität des Chromatins nicht nur von der gesamten Kernsubstanzmasse, sondern auch von der Anzahl der Kernelemente, auf welche Kernsubstanz verteilt ist, abhängt.

Nun drängt sich die Frage auf, ob 1) die gesamte Kernsubstanzquantität in denselben Entwicklungsstadien immer konstant ist und ob 2) der gesamte Chromatingebalt keinen Schwankangen unterliegt. In einer solchen Analyse müssen die Einflisse äußerer und innerer Faktoren untersucht werden. Daß die Keruplasmarelation in einzelnen Zellen eines bestimmten Entwicklungsstadiums bei denselben Tierspecies von den äußeren Bedingungen in gewissem Grade abhängig ist, geht aus der Arbeit von Marcus (06) hervor. Seine Experimente hatten den $Z$ weck, festzustellen, inwiefern die quantitative Kernplasmarelation durch die Temperatur, in welcher die Entwicklung verläuft, beeinflußt wird. Er kommt auf Grund der Bestimmung der 
Kernplasmarelation in Kulturen, welche bei drei verschiedenen Temperaturen geztichtet wurden, zu dem Schluß:

*Es existiert somit eine Beeinflussung der Kernplasmarelation durch die Temperatur, und zwar in der Kälte zu ungunsten des Plasmas.* Ich habe ähnliche, iberdies aber anch andre Versuche angestellt, um mich zu überzengen, ob die Art der Kernsubstanzproduktion von äußeren Faktoren beeinflußt wird. Meine Experimente mit der Züchtung der Kulturen in versehiedenen Temperaturen ergaben bis zum Blastulastadium eine Bestätigung der Marccsschen Resultate.

Die befruchteten Echinus- und Sphaerechinus-Eier wurden in zwei verschiedenen Temperaturen, nämlich bei $12^{\circ}$ und $21^{\circ}$, gehalten. Die Blastulae wurden am Anfang der Mesenchymbildung fixiert und an der Hand der gefärbten Präparate die Kernsubstanzquantität nach den oben beschriebenen Methoden bestimmt.

Auf Grund dieser Angaben konnte die absolute Kernsubstanzmenge festgestellt werden. Die Befunde, welche sich auf die aus zwei verschiedenen Temperaturen herstammenden Objekte beziehen, stellte ich in der unten angegebenen Tabelle zusammen.

Tabelle II.

\begin{tabular}{|c|c|c|c|c|c|}
\hline $\begin{array}{c}\text { Tempera- } \\
\text { tur }\end{array}$ & $\begin{array}{l}\text { Zahl der Kerne } \\
\text { am } \\
\text { Blastulaumfange }\end{array}$ & $\begin{array}{c}\text { Die Zahl der } \\
\text { Zellen, aus wel- } \\
\text { ehen die Blastula- } \\
\text { wand zusammen- } \\
\text { gresetzt ist }\end{array}$ & $\begin{array}{c}\text { Kern- } \\
\text { durchmesser } \\
\text { (Mittelwert }\left.\right|^{1} \text { ) }\end{array}$ & $\begin{array}{c}\text { Kern- } \\
\text { volumen }\end{array}$ & $\begin{array}{c}\text { Absolute Krrn- } \\
\text { sabstanzmenge } \\
\text { der } \\
\text { Blastulawand }\end{array}$ \\
\hline $12^{\circ}$ & $\begin{array}{c}54,57,54,59,54 \\
57,57,56,56,55 \\
\text { Mittelwert }=56\end{array}$ & 809 & 4,2 & 38,8 & 31417 \\
\hline $21^{\circ}$ & $\begin{array}{c}68,66,70,64,68 \\
70,64,70,65.70 \\
\text { Mittelwert }=68\end{array}$ & 1467 & 3,4 & 20,6 & 30276 \\
\hline
\end{tabular}

Aus den in dieser Tabelle angeführten Ziffern ist ersichtlich, daß die Temperatur die Art der Verteilung auf größere oder kleinere Kernenzahl beeinflußt. Auf Grund dieser Ergebnisse kann ich also die Befunde von Marces bestätigen. In Übereinstimmung mit dem

1) Die Berechnungen wurden auf Grund der MIessungen nach dem Millimetermaßstab anf den Zeichnungen, die mittels der Zsiss-Camera, Immers. $2 \mathrm{~mm}$. K.OK. 6, Tubusl. 155, entworfen wurden, ausgeführt. Die relativen Größen sind in Millimetern ausgedrïckt und beziehen sich nicht auf das Objekt selbst, sondern auf die Zeichnung (1000 fache Yergrößerung des Objekts). 
genannten Autor kann man aus den angeführten Ziffern den Schluß ziehen, daß die Anzahl der Kerne bzw. der Zellen, aus welchen die Blastulawand sich zusammensetzt, größer ist, wenn die Eier bei hüherer Temperatur sich entwickelt haben, und daß im Gegenteil die bei niederer Temperatur gezuichteten Blastulae aus einer kleineren Zellenzahl bestehen. In Übereinstimmung mit den Befunden von Marces zeigt unsre Tabelle, weiter, daß die Kerne der Blastulae, welche in höherer Temperatur gezüchtet wurden, kleiner sind als die Kerne der in niedrigerer Temperatur kultivierten Embryonen desselben Entwicklungsstadiums. Aus der Tabelle ist sodann zu ersehen, daß bezüglich der absoluten Kernsubstanzmenge, welche in beiden Kulturen während der Entwicklung bis zum Blastulastadium produziert wurde, kein beträehtlicher Unterschied besteht, da ja der vorhandene wahrscheinlich noch innerhalb der bei der Skizzierung wohl möglichen Fehlergrenzen liegt.

Es drängt sich nun die Frage auf, ob die Art der Kernsubstanzverteilung auch von andern äußeren Faktoren abhängen kann. Ich habe also zunächst Versuche angestellt, um zu prüfen, ob die Konzentration des äußeren Mediums einen Einfluß auf den Prozeß der Kernsubstanzproduktion, bzw. auf die Art der Verteilung desselben haben kann.

Aus der Arbeit von Driesch (93) ist bekannt, daß die Seeigeleier auch in verdïnntem Seewasser sich entwickeln können. DrIEsch hat dabei festgestellt, daß man durch Verdünnung des Seewassers *konstante Abweichungen in den Dimensionen der Zellen « hervorrufen kann. Sodann beobachtete Driesch hierbei eine Verlangsamung der Zellteilung, ja sogar die Kernteilung ohne Zellenteilung, so daß daraus mehrkernige Zellen resultierten. Auch das vorzeitige Hervortreten der Micromeren ist beobachtet worden; trotzdem haben die Eier, welche sich in diesen Verhältnissen entwickelt haben, durchaus normale Plutei gebildet.

Bei einem Teil meiner Versuche habe ich das natürliche Seewasser mit destilliertem Wasser verduinnt, bei einem andern Teil habe ich die befruchteten Eier in Seewasser von höherer Konzentration gebracht, indem ich zu normalem künstlich bereitetes Seewasser von doppelter Konzentration zugesetzt habe.

Im Blastulastadium, am Anfang der Mesenchymbildung, wurden die Embryonen der einzelnen Kulturen fixiert und die Untersuchung auf die oben geschilderte Weise durchgeführt. Die beistehende Tabelle III enthält die Resultate dieser Versuchsreihe. 
Tabelle III.

\begin{tabular}{|c|c|c|c|c|c|}
\hline $\begin{array}{l}\text { Die Konzentration } \\
\text { des Seewassers }\end{array}$ & $\begin{array}{l}\text { Die Zahl der herme } \\
\text { am Blastulaumfang }\end{array}$ & $\begin{array}{l}\text { Die Gesamt- } \\
\text { zahl } \\
\text { der Zellen }\end{array}$ & $\left\{\begin{array}{c}\text { Der Durch- } \\
\text { messer } \\
\text { des herns } \\
\text { (Nittelwert) }\end{array}\right.$ & \begin{tabular}{|c|} 
Durch- \\
schnittliches \\
Volumen \\
des Kerns
\end{tabular} & $\begin{array}{l}\text { Gesamte } \\
\text { Kirn- } \\
\text { substanz- } \\
\text { meng:- }\end{array}$ \\
\hline $\begin{array}{c}40 \text { Seewasser : } 10 \\
\text { destilliertes Wasser }\end{array}$ & $\begin{array}{l}60,61,63,61,62, \\
59,59,59,62,61, \\
\quad \text { im Mittel } 60\end{array}$ & 902 & 4,3 & 41,67 & 37501 \\
\hline normal & $\begin{array}{l}65,59,62,64,63 \\
65,68,69,67,66 \\
\quad \text { im Mittel } \mathbf{6 5}\end{array}$ & 1331 & 3.6 & 24.40 & 32551 \\
\hline $\begin{array}{c}45 \text { normales See- } \\
\text { wasser : } 5 \text { Seewass. } \\
\text { von doppelter Kon- } \\
\text { zentration }\end{array}$ & $\begin{array}{l}78,72,69,76,76 \\
78,72,73,72,69 \\
\quad \text { im Mittel } 75\end{array}$ & 1781 & 3,3 & 18.49 & 32926 \\
\hline
\end{tabular}

An der Hand dieser Tabelle läßt sich wieder feststellen, daß die Anzahl der Kerne, auf welche die während der Entwicklung ansgebildete Kernsubstanzmasse verteilt ist, anch von der Konzentration des umgebenden Seewassers abhängig ist.

Wir sehen nämlich, daß die Blastulae, welche sich aus den in rerdünntem Seewasser kultivierten Eiern entwickelt haben, aus einer kleineren Zellenzahl bestehen als diejenigen, welche in normalem Seewasser gezüchtet wurden. Ḋer Unterschied ist noch beträchtlicher, wenn man zum Vergleich die in verdünntem und in konzentriertem Seewasser kultivierten Blastulae heranzieht. In konzentriertem Seewasser gezüchtete Blastulae bestehen aus einer bedeutend größeren Zellenzahl als diejenigen, welche in verduinntem Seewasser sich entwickelt haben. Umgekehrt verhalten sich aber die Kerngrößen. Die Dimensionen der Kerne sind die größten, wenn die Embryonen sich in verdüntem Seewasser entwickelten, mittelgroß sind sie in der Kultur aus normalem Seewasser, am kleinsten sind die Kernvolumina, wenn die Entwicklung in konzentriertem Seewasser vor sich ging. Was den allgemeinen Kernsubstanzgehalt betrifft, so scheinen mir die in der Tabelle verzeichneten Differenzen zwischen den einzelnen Kulturen nicht sehr beträchtlich zu sein und es erscheint auch hier der Schluß berechtigt, daß durch die Einwirkung äußerer Faktoren eigentlich nur die Verteilungsweise, nicht aber die Quantität der gesamten Kernsubstanzmasse beeinflubt wird.

In der dritten Versuchsserie habe ich den EinfluB der Konzentration der OH-Ionen im Seewasser auf die Kernsubstanz- 
produktion geprift. Es war nämlich aus zahlreichen Literaturangaben der neueren Zeit bekannt, daß die OH-Ionen für die Entwicklung von großer Bedeutung sind. HERBst hat im Jahre 1898 die Notwendigkeit eines bestimmten Alkalinitätsgrades des umgebenden $\mathrm{Me}-$ diums für die normale Entwicklung der Seeigellarven nachgewiesen. In demselben Jahre hat Loeb darauf hingewiesen, daß die Embryonalentwicklung von Arbacia beschleunigt werden kann, wenn man die Alkalinität des Seewassers durch Zusatz von $\mathrm{NaOH}$ erhöht. In einer eingehenden Studie hat im Jahre 1904 Herbst weitere Beweise für die Unentbehrlichkeit von OH-Ionen für die Entwicklung der Seeigel erbracht. Wenn man auch die bekannten Arbeiten von LoEB ${ }^{1}$ ) über die Bedeutung der OH-Ionen für die Bastardierungsversuche bericksichtigt, so geht aus alledem obne weiteres hervor, daß der Gehalt an $\mathrm{OH}-\mathrm{I}$ onen im umgebenden Medium für die biologischen Prozesse der Entwicklung von prinzipieller Bedeutung ist.

Die Experimente von Herbst (04) haben ergeben, daß die Menge der OH-Ionen im Seewasser des Golfes von Neapel für die Echinusund Sphaerechinus-Entwicklung die optimale ist. Durch Zusatz verschiedener Mengen von $1 / 10 n$ Essigsäure habe ich die Konzentration der OH-Ionen im Seewasser herabgesetzt, durch Zusatz von ${ }_{1}^{\prime}{ }_{10} n \mathrm{NaOH}$ habe ich in andern Experimenten diese Konzentration erhöht. Die Probeversuche ergaben, daß durch einen Zusatz von $0,4 \mathrm{~cm} 1 / 10$ nssigsäure zu $100 \mathrm{ccm}$ Seewasser die Entwicklung noch nicht beeinträchtigt wurde. Bei Gelegenheit meiner Bastardierungsarbeit (06) habe ich in Neapel mich überzeugt, daß man bei Zusatz ron $1 \mathrm{cem} 1 / 10 n \mathrm{NaOH}$ auf $100 \mathrm{ccm}$ Seewasser ebenfalls auf normale Entwicklung der Seeigel rechnen kann. Die Kulturen, welche unter diesen Bedingungen sich entwickelten, wurden im Blastulastadium auf ihre Zellenzahl und ihre Kerngröße in der oben beschriebenen Weise geprüft.

Die Ergebnisse dieser Untersuchungen sind in der beistehenden Tabelle IV (S. 298) zusammengestellt.

Aus den Ziffern dieser Tabelle kann der Schluß gezogen werden, daß die Konzentration der OH-Ionen im umgebenden Medium die Art der Kernsubstanzproduktion während der Entwicklung der Seeigel beeinflussen kann. Die Ziffern beweisen nämlich, daß bei der Herabsetzung der $\mathrm{OH}-$ Ionen-Konzentration die Blastulae aus einer kleineren Zellenzahi zusammengesetzt sind als diejenigen, welche in normalem

1) Vergleiche Literaturrerzeichnis meiner Bastardierungsarbeit 06 , 
Tabelle IV.

\begin{tabular}{|c|c|c|c|c|c|}
\hline $\begin{array}{c}\text { Die Flüssigkeit, } \\
\text { in weleher die Entwick- } \\
\text { lung rerlief }\end{array}$ & $\begin{array}{l}\text { Die Zahl der Kerne } \\
\text { am Blastulaumfang }\end{array}$ & $\begin{array}{c}\text { Die Gromt- } \\
\text { zahl } \\
\text { der } \\
\text { Zellen }\end{array}$ & $\begin{array}{l}\text { Der Durch- } \\
\text { messer } \\
\text { des herns } \\
\text { (Mittelwert) }\end{array}$ & $\begin{array}{l}\text { Volumen } \\
\text { des } \\
\text { herns }\end{array}$ & $\begin{array}{c}\text { Grisante } \\
\text { Kern- } \\
\text { substanz- } \\
\text { menge }\end{array}$ \\
\hline $\begin{array}{c}100 \mathrm{ccm} \text { Seewasser } \\
+0.4 \mathrm{ccm} 1 / 10 n \\
\text { Essigsäure }\end{array}$ & 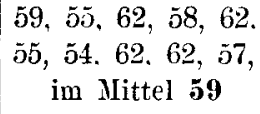 & 1138 & 3,8 & 28,76 & 32730 \\
\hline $\begin{array}{c}\text { gewöhnliches See- } \\
\text { wasser }\end{array}$ & $\begin{array}{l}62,67,59,63,63 \\
65,65,58,61.63 \\
\text { im Mittel } \mathbf{6 2}\end{array}$ & 1256 & 3,6 & $24,4 \tilde{5}$ & 30716 \\
\hline $\begin{array}{c}100 \mathrm{ccm} \text { Seewasser } \\
+1 \mathrm{~cm} 1 / 10 n \\
\mathrm{NaOH}\end{array}$ & $\begin{array}{c}70,77,79,76,70 \\
75,78,80,79,80 \\
\quad \text { im Mittel } 76\end{array}$ & 1841 & 3,0 & 14,12 & 26114 \\
\hline
\end{tabular}

Seewasser ihre Entwicklung durchgemacht haben. Der Zusatz von $\mathrm{NaOH}$ zum Seewasser, also die Erhöbung der Alkalinität, veranlaßt dagegen öftere Zellteilungen, so daß die Blastulae solcher Kulturen ans einer größeren Zellenzahl bestehen. Umgekehrt verhält sich die Kerngröße. Im Seewasser, dessen Alkalinität herabgesetzt wurde, entwickeln sich Blastulae, deren Zellkerne größer sind als diejenigen der im gewöhnlichen Seewasser gezüchteten Embryonen. Am kleinsten sind die Kerne jener Blastulae, welche sich im Seewasser von erhöhter Konzentration der Hydroxylionen entwickelt haben.

Vergleicht man endlich die gesamte Kernsubstanzmenge, welche während der Entwicklung der Individuen einzelner Kulturen produziert wurde, so ersieht man, daß hier der Unterschied in einzelnen Kulturen etwas größer ist als in den vorher beschriebenen Experimentenserien. Jedoch bleibt auch hier dasselbe Prinzip; das, was der Embryo an Größe der Kerne verliert, sucht er an der Zahl derselben zu gewinnen, so daß sich diese zwei Momente wenigstens bis zu einem gewissen Grade kompensieren.

Es stellt sich demnach heraus, daß sowohl die Temperaturveränderungen, als auch die Veränderungen der Konzentration des Seewassers, endlich auch der veränderte Gehalt der OH-Ionen im umgebenden $\mathrm{Me}$ dium die Quantität der produzierten absoluten Kernsubstanzmenge in größerem Maße weder beeinträchtigen noch fördern können. Die absolute Kernsubstanzmasse bleibt, von verbältnismäßig unbedeutenden Schwankungen abgesehen, von den äußeren Einflïssen unabhängig, fast konstant. Dagegen werden bezîglich der Produktion der Kern- 
substanz zwei Momente, die eng miteinander zusammenhängen, von den äußeren Faktoren tatsächlich beeinflußt, und zwar 1) die Verteilung der Kernsubstanz, 2) der Chromatingehalt in der absoluten Kernsubstanzmenge.

Wir haben gesehen, daß sich dieselbe Kernsubstanzmasse auf eine kleinere oder größere Anzahl von Kernen verteilen kann. Ich glaube im vorhergehenden Kapitel nachgewiesen zu haben, daß während der zweiten Furchungsperiode durch die aufeinander folgenden Kernteilungen sich die Kernsubstanz an Chromatin bereichert. Je mehr solcher Teilungen während der zweiten Furchungsperiode stattgefunden haben, desto reicher wird die absolute Kernsubstanzmasse an Chromatin, vorausgesetzt, daß die Größe der Chromosomen konstant bleibt ${ }^{1}$.

Die erhöhte Temperatur, die größere Konzentration des Seewassers und der erhöhte OH-Ionen-Gehalt im umgebenden Medium. haben zur Folge, daß die Kernsubstanzmasse im Blastulastadium auf eine größere Anzahl von Kernen verteilt ist. In dieser Tatsache liegt ein Beweis dafür, daß die Zahl der durchgemachten Kernteilungen größer war und daß eben infolgedessen der embryonale Organismus auf demselben Entwicklungsstadium größere Chromatinmenge besitzt. Im Gegenteil wird die Häufigkeit der Kernteilung von der niedrigeren Temperatur, der niedrigeren Salzkonzentration im Seewasser und dem niedrigeren Alkalinitätsgrad desselben beeinträchtigt. Obschon sich also dieselbe Quantität an Kernsubstanz während der Entwicklung anbäuft, ist sie doch nicht so reich an Chromatin.

Aus dem Gesagten können wir somit den allgemeinen Schluß ziehen: die Menge der produzierten Kernsubstanz ist von den äußeren Faktoren fast unabhängig, die Produktion der absoluten Chromatinquantität wird dagegen von äußeren Bedingungen beeinfluBt1).

In den bisherigen Erörterungen haben wir den Einfluß der äußeren Faktoren auf die Kernsubstanzproduktion während der Furchungsprozesse bis zum Blastulastadium besprochen. Da ich in dem vorhergehenden Kapitel darauf hingewiesen habe, daß auch in späteren Entwicklungsstadien die Kernsubstanzproduktion vor sich geht, so drängt sich die Frage auf, ob dieser Prozeß auch in späterer Entwicklung von äußeren Faktoren beeinflußt werden kann.

1: Vergleiche Nachtrag zu dieser Arbeit S. 328. 
Marcus (06), welcher, wie oben erwähnt wurde, den Einfluß der Temperatur auf die Kernplasmarelation untersueht hat, führt in seiner Tabelle II Ziffern an, nach welchen der Unterschied im Kerndiameter der »Kälte- und der Zimmerkultur « sich schon im Stadium des armlosen Plutens ausgleichen soll. Diesen Befund von Marcus kann ich auf Grund meiner Untersuchungen nicht bestätigen. Die Kerne sind im Pluteusstadium oft etwas kleiner als die Kerne derselben Kultur im Blastulastadium. Diese Verkleinerung ist jedoch nicht konstant. Ich habe mehrmals Kulturen beobachtet, in welchen die Kerndimensionen zwischen dem Blastula- und Pluteusstadium an Größe überhaupt nicht abgenommen haben, oder sogar noch etwas größer gewesen sind. Über den Temperatureinfluß auf die Größe der Plnteuskerne können wir uns auf Grtnd der Fig. 8 orientieren. Fig. $8 a$ stellt die Größe der Kerne von Plutei dar, welche in der Temperatur von $12^{\circ}$ gezuichtet wurden, auf der Fig. $8 l$ sind wieder die Kerne aufgezeichnet, welche aus der Kultur der Plutei herstammen, die in der Temperatur von $21^{\circ}$ sich entwickelt haben. Der Unterschied fällt sofort in die Augen. Ja, ich kann sogar aus meinen Beobachtungen den Schluß ziehen, daß die im Blastulastadium notierten Differenzen sich in weiterer Entwicklung noch mehr erhöhen.

Ich habe den Untersehied in der Kerngröße der Plutei, welche in rerschiedenen Seewasserkonzentrationen gezuichtet wurden, auch feststellen können: er war im Pluteusstadium nicht kleiner als in der Blastula. Minder dentlich und minder konstant waren die Differenzen in der Kerngröße der Plutei, welche sich im Seewasser von verschiedenem Gehalt an $\mathrm{OH}-$ Ionen entwickelten.

Wir haben also festgestellt, daß die Größen der einzelnen Kerne durch die äußeren Faktoren beeinflußt werden können. Ob dadurch die absolute Quantität der gesamten Kernsubstanzmenge beeinflußt wird, läßt sich nicht leicht entscheiden, da die genanere Bestimmung der Kernenanzahl sehr schwer durchfuhrbar wäre.

Aber doch scheinen mir diese Tatsachen beachtenswert zu sein. Bekanntlich hat Boveri (05) in seiner grundlegenden Arbeit die These aufgestellt: „Es sind also die Oberflächen der Kerne ihrer Chromosomenanzahl und damit auch der in ihnen enthaltenen Chromatinmenge direkt proportional. « Wenn man anf Grund dieser so wichtigen Regel die Genese der Kerne beurteilt, so soll man immer auch die außeren Faktoren der Entwicklung beriicksichtigen, da wir gesehen haben, daß die Kerngrüße noch im Pluteusstadium auch von den äußeren Faktoren beeinflußt werden kamn. 
Und diese Tatsache muß besonders in den Studien über das Vererbungsproblem berücksichtigt werden. Bekanntlich sind bisher alle Vererbungsforschungen, welche auf dem Gebiete der experimentellen Cytologie entstanden sind, iber das Lokalisationsproblem leider nicht hinausgegangen. Bei diesen Untersuchungen, welche sich hauptsächlich auf die von Boveri aufgestellten Regeln stützen, spielt die Beurteilung der Kerngrößen der Versuchslarven oft eine prinzipielle Rolle (vgl. die Arbeiten von Boveri, Godlewski jun., Herbst). Der Einfluß der äußeren Faktoren muß also bei dieser Untersuchungsmethode auch berücksichtigt werden.

Die Hauptergebnisse dieses Kapitels lassen sich also folgendermaßen zusammenstellen:

1) Die erhöhte Temperatur, die größere Konzentration der Salze im Seewasser, der höhere Alkalinitätsgrad des umgebenden Mediums begiunstigen die Häufigkeit der Zellteilungen während der Furchung. Infolgedessen sind die Keime, welche sich in diesen Bedingungen entwickelt haben, im Blastulastadium aus größerer Zellenanzahl zusammengesetzt als die Blastnlae, welche in niedrigerer Temperatur, in verdünntem Seewasser, oder bei niedrigerem Alkalinitätsgrad gezüchtet wurden.

2) Da in der zweiten Furchungsperiode keine beträchtlichere Zunahme der absoluten Kernsubstanzmasse des Keimes stattfindet und durch die Teilungen dieser Furchungsperiode die früher produzierte Kernsubstanzmasse auf größere Kernenanzahl verteilt wird, so sind die Kerne jener Blastulae, welche sich in niedrigerer Temperatur, geringerer Salz- und OH-Ionen-Konzentration entwickelt haben, kleiner als diejenigen, welche in der Kälte, in verdünntem Seewasser, oder vermindertem Alkalinitätsgrad ihre Entwicklung durchgemacht haben und aus kleinerer Zellenanzahl zusammengesetzt sind.

3) Die absolute Kernsubstanzmenge des Keimes scheint von den äußeren Faktoren unabhängig zu sein.

4) Die absolute Chromatinquantität kann von der Temperatur, von der Konzentration des Seewassers und vom Alkalinitätsgrad beeinflußt werden 1 ).

5) Die Abhängigkeit der Kerngröße von den äußeren Faktoren kann noch im Pluteusstadium walınehmbar sein.

1: Vergleiche Nachtrag zu dicser Arbeit S. 328. 


\section{Teil.}

\section{Der Verlauf der Entwicklung und der Kernsubstanzbildung bei der durch $\mathrm{CO}_{2}$-haltiges Seewasser gehemmten Plasmateilung.}

Die Bildung der Kernsubstanz tritt mit der größten Intensität in den ersten Entwicklungsphasen auf und läßt sich während der Furchung besonders dentlich in den successiven Furchungsstadien verfolgen. Die Kernvermehrung begleitet bei dem gewöhnlichen Furchungsverlauf die Zellleibsteilung. Aus den Arbeiten von Loeb 195 , Driesch $(92,95)$, Wilson (01), Lillie (01, 05), Scott (06), KostaNECKI (08) u. a. ist jedoch bekannt geworden, daß die Kernteilung ohne Zellteilung während der Entwicklung vor sich gehen kann, so daß daraus mehrkernige Eier resultieren. Es erhebt sich also bei der Erwägung dieser Tatsache die Frage, ob die Furchung bzw. die Plasmateilung, welche in der Regel die embryonalen Kernteilungen begleitet, als ein die Kernsubstanzproduktion regulierender Faktor aufzufassen ist, oder ob diese beiden Vorgänge unabhängig voneinander verlaufen können.

Im Nachfolgenden möchte ich über meine Versuche berichten, welche behufs Entscheidung dieser Frage angestellt wurden. Ich habe meine diesbezüglichen Experimente derart angestellt, daß ich die Plasmateilung der Echinus-Eier durch Einwirkung von $\mathrm{CO}_{2}$-haltigem Seewasser, oder durch Anwendung hypertonischer Lösungen gehemmt habe, wobei jedoch die Kernteilung nicht beeinträchtigt wurde. Wenn auch manche Details, welche ich bei diesen Experimenten beobachtet habe, nur in weiterem Zusammenhange mit dem uns vor allem beschäftigenden Problem stehen, sollen sie doch hier mit beriicksichtigt werden.

1. Die Methode und Untersuchungsaufgabe.

Wie oben erwähnt, habe ich die Echinus-Eier mit $\mathrm{CO}_{2}$-haltigem Seewasser behandelt. Die Methode der Kohlensäureeinwirkung auf die Eier wurde bekanntlich von DeLage (02) zuerst behufs der Herrorrufung der künstlichen Parthenogenese bei Asteriden in die experimentelle Cytologie eingefithrt. In meinen Experimenten habe ich Meerwasser verwendet, welches vollkommen oder nur teilweise mit Kohlensäure gesättigt wurde. Zur Sättigung des Seewassers benutzte ich den KIPPschen Apparat. Die durch ein Gefäß mit $\mathrm{NaHCO}_{3}$-Lösung durchgeleitete Kohlensäure wurde durch die mit Seewasser gefüllte Flasche einige 
Stunden lang durchgeleitet. In einer Versuchsreihe wurden die Eier in dieses mit $\mathrm{CO}_{2}$ gesättigtes Wasser auf 2-7 Minuten gebracht. In anderu Experimenten wurde das mit $\mathrm{CO}_{2}$ gesättigte Seewasser mit gewöhnlichem Seewasser in verschiedenen Verhältnissen verdünnt und die Eier erst mit dieser Mischung behandelt; bei dem letaten Verfahren verblieben die Eier etwa 6 Stunden in der Mischung. Behufs weiterer Ziichtung wurden sie nach mehrmaliger Ausspiilung in gewöhnliches Seewasser übertragen. Bie Behandlung mit dem $\mathrm{CO}_{2}$ haltigen Seewasser betraf in einer Versuchsserie unbefruchtete Eier, so daß die Befruchtung erst nach der Ausspuilung der Eier unternommen wurde. In andern Experimenten wurden vorher befruchtete, schon in Entwicklung begriffene Eier der Einwirkung von $\mathrm{CO}_{2}$-haltigem Seewasser ausgesetzt und zwar entweder unmittelbar vor dem Auftreten der ersten Furche, oder im Zwei- bis Vier-, oder Achtblastomerenstadium. Außerdem habe ich mich auch der hypertonischen Lösungen bedient, welche gewöhnlich zur Hervorrufung der kiinstlichen Parthenogenese gebraucht werden (besonders $2 \frac{1}{2} n \mathrm{KCl}$ ).

Die Untersuchung bezweckte erstens, die Veränderungen, welche unter dem Einfluß des veränderten Mediums hervortreten, kennen zu lernen, sodann die Regulationsvorgänge, welche den normalen Zustand in der Organisation herzustellen suchen, zu erforschen, endlich - und dies habe ich besonders berücksichtigt - die Transformation des Protoplasmas in Kernsubstanz in dem sich ohne Zellteilung entwickelnden embryonalen Organismus mit dem normal verlaufenden Furchungsprozess zu vergleichen.

Die zwei ersten Aufgaben konnten am lebenden Material teilweise gelöst werden, den Transformationsprozeß des Protoplasmas in Kernsubstanz konnte man natürlich nur am fixierten Material feststellen.

2. Die Veränderungen in den äußeren Befruchtungserscheinungen unter dem Einfluß des $\mathrm{CO}_{2}$-haltigen Seewassers.

Wenn die Eier aus dem mit $\mathrm{CO}_{2}$ gesättigten in normales Seewasser heribergebracht wurden, so ergab sich, daß bei den Eiern, welche kürzer als 2-3 Minuten im $\mathrm{CO}_{2}$-haltigen Seewasser geblieben sind, die Befruchtungsfähigkeit in keiner Weise beeinträchtigt wurde. Auch ihre Entwicklungsfähigkeit ist vollkommen normal geblieben. Werden die unbefruchteten Eier der Einwirkung von $\mathrm{CO}_{2}$ auf 3 bis $4 \frac{1}{2}$ Minuten ausgesetzt, so bilden sie nach der Übertragung in nor- 
males Seewasser die charakteristische Befruchtungsmembran. Diese Tatsache wurde schon von J. LOEB (05) für Strongylocentrotus purpuratus festgestellt. Die künstliche Hervorrufung der Membran an unbefruchteten Eiern gelingt in Neapel mit dem $\mathrm{CO}_{2}$-haltigen Seewasser bedeutend leichter als mit der andern auch von J. LoEb (05) angegebenen Fettsäuremethode.

Die Anzahl der Eier, welche nach der Behandlung mit dem $\mathrm{CO}_{2}$ haltigen Seewasser die Dottermembran gebildet haben, war sehr verschieden. Nur äußerst selten habe ich ein Weibchen gehabt, dessen Eier fast alle durch die Kohlensäureeinwirkung zur Membranerzeugung angeregt werden konnten 1 ).

Ich habe nun versucht, die Eier, welche die künstliche Membran gebildet haben, zu befruchten. Zu diesem Zwecke habe ich die Eier, auf welchen die Membranbildung künstlich hervorgerufen wurde, herausgefischt, isoliert, und zu ihnen Sperma hinzugefügt. Die Entwicklung hat, wenn sie uiberhaupt begann, das Zweizellenstadium niemals iiberschritten. Der Anstoß zur Parthenogenese, welcher vom $\mathrm{CO}_{2}$-haltigen Seewasser den Eiern zugebracht wurde und welcher in

1: In dieser Tatsache sehe ich den neuen Beweis, daß die Individualität der Weibchen hier eine äußerst wichtige Rolle spielt. Es ist allgemein bekannt, daß sich das Material, welches in den europiiischen zoologischen Stationen zur Verfiigung steht, tür die Hervorrufung der künstlichen Parthenogenese bedeutend schlechter eignet als das amerikanische Material. Dasselbe kann man auch von der künstlichen Hervorrufung der Dottermembran sagen. Alle bisher in Europa angewandten Nethoden der künstlichen Erzeugung der Befruchtungsmembran haben quantitativ lange nicht die Resultate erreicht, welche die Untersuchungen der amerikanischen Forscher ergeben haben. J. LOEB (0) gibt in seinen Mitteilungen über die verbesserte Methode der kïnstlichen Parthenogenese an, daß die Eier, welche zur künstlichen Membranbildung angeregt wurden, durch denselben Reiz auch einen schwachen Anstoß zur künstlichen Parthenogenese bekommen. Durch die Kombination dieser Fettsïuremethode und der Behandlungen mit den hypertonischen Lösungen hat LoEB fast 100\% normal sich entwickelnder Embryonen bekommen. In Übereinstimmung mit Hersst :06, habe ich mich iiberzengt, daß, obschon die Fettsäuremethode sich zur Hervorrufung der künstlichen Membranen am Neapler Material nicht so gut eiguet, wie es am amerikanischen Material konstatiert wurde, immerhin den Eiern durch dieses Verfahren ein leichter Anstof zur Parthenogenese gegeben wird; die Entwicklung iiberschreitet jedoch nie das Zweizellenstadium. Auch die Kombination der Fettsizurebehandlung und der Einwirkung der hypertonischen Lüsungen hat keine besonders günstigen Resultate ergeben. Ich habe mehrmals in Neapel und Triest diese Methode versucht. Wenn auch durch die Kumulation der Reize die parthenogenetische Entwicklung bei einer kleinen Anzahl begonnen hat, so war am Neapler oder 'Triestiner Material der Entwicklungsverlauf gar nicht so regelmïßig, wie ihn LoEs in Kalifornia beobachtet hat. 
den Experimenten ron Delage (02) ausreichte, um die weit fortgeschrittene parthenogenetische Entwicklung der Seesterneier hervorzurufen, war zu schwach, um die Echinus-Eier zu weiterer Entwicklung anzuregen. Aus dem Versuche ist weiter zu ersehen, daß auch das den Eieru zugefügte Sperma die Entwicklung nicht zu veranlassen vermochte. Aller Wahrscheinlichkeit nach sind die Spermatozoen in das Ei nicht eingedrungen. Diese Tatsache könnte als Ausdruck des allgemein anerkannten Prinzips betrachtet werden, da $B$ die Spermatozoen durch die Dottermembran nicht hindurchdringen können. Um jedoch daruber ein Urteil zu gewinnen, habe ich die Eier, auf welchen die künstliche Membran erzeugt wurde, isoliert, durch Schütteln von der künstlichen Membran befreit, in frisches Seewasser herübergebracht und zu solchen Eiern Sperma hinzugefügt. In zwei von mir derart angestellten Experimenten hat sich kein einziges Ei entwickelt, allem Anschein nach sind also die Spermatozoen in diese Eier nicht eingedrungen, obschon die künstlich erzeugte Dottermembran abgeschüttelt wurde.

Daraus könnte der Schluß gezogen werden, daß nicht nur die Dottermembran, sondern auch andre Faktoren das Eindringen der Spermatozoen in solche Eier verhindern. Diese Tatsache scheint mir auch aus diesem Grunde ron Bedeutung zu sein, da sie mit der Frage der Verhütung der Polyspermie bei dem gewöhnlichen Befruchtung:verlauf im Zusammenhang steht.

In der Beurteilung der Momente, welche die Eier vor der Polyspermie schützen, ist auch die andre wohlbekannte Erscheinung zu beachten: die Eier, welche nach der Befruchtung die Dottermembran erzeugen und nachher mit der Methode von Driesch von ihr durch Schütteln befreit werden, können eine ganz normale Entwicklung. durchmachen, obschon sie in dem spermahaltigen Wasser weiter verbleiben. Die Polyspermie ist hier also nicht eingetreten, obschon die Membran beseitigt wurde und die Samenfäden den freien Zutritt zo den Eiern gehabt hatten.

Diese Versuchsergebnisse sind meiner Ansicht nach dahin zu deuten, daß die Befruchtungsmembran nicht als einziger Faktor, welcher der Polyspermie vorzubeugen imstande ist, betrachtet werden kann. Möglicherweise beginnen gleichzeitig mit der Bildung der Membran im Ei auch innere Veränderungen ${ }^{1}$ ), welche das Ein-

1) DRIEsch hat bekanntlich nachgewiesen, daß die physikalischen Eigenschaften des Eiprotoplasmas, besonders der Aggregatzustand desselben, gleich nach dem Eindringen des Spermatozoons gewisse Veränderungen erfihrt. Ich 
dringen der Spermatozoen bzw. die wiederholte Befruchtung unmöglich machen.

Im Vorhergehenden haben wir also erfahren, daB mit $\mathrm{CO}_{2}$ gesättigtes Meerwasser die Membranbildung hervorrufen kann, wenn es $2-4_{1}^{1}, 2$ Minuten auf die Eier einwirkt. Diese Eier ließen sich auch nach der Beseitigung der Membran nicht befruchten.

Bleiben die Eier 6-12 Minuten im Seewasser, welches mit $\mathrm{CO}_{2}$ gesättigt wurde, und werden sie nachber in gewöhnliches Seewasser tubertragen, so bilden sie nie die Dottermembran. Setzt man zu solchen Eiern Sperma hinzu, so stellt sich bald heraus, daß die Eier die Befruchtungsfähigkeit, ebensowenig die Entwicklungsfähigkeit, nicht eingebüßt haben, obschon ihr Entwicklungsverlauf verändert ist ${ }^{1}$.

Von 0 . und R. Hertwig (87) wurde festgestellt, daß die Eier, welche längere Zeit im Seewasser gelegen haben und erst später befruchtet worden sind, keine Befruchtungsmembran bilden, daß aber ihre Entwicklungsfähigkeit dadurch nicht beeinträchtigt wird. Diese Beobachtung wurde nachher von Boveri bestätigt, ich habe auch oftmals Gelegenheit gehabt, solche membranlose Eier sich entwickeln zu sehen. Es muß hervorgehoben werden, daß es ron der Indiridualität der Eier abhängt, wie lange dieselben vor der Befruchtung in Seewasser liegen miissen, bis sie die Membranbildungsfähigkeit einbiißen. Läßt man die Eier 6-12 Stunden im Seewasser liegen, so bildet oft kein Ei nach der Besamung die Dotterhaut; in andern Fällen könuen die Eier 24 Stunden im Seewasser verbleiben und doch exzeugen sie sofort nach der Befruchtung die Membran.

Es drängt sich bei der Erwägung der beschriebenen Tatsachen die Frage auf, ob das Einbüßen der Fïhigkeit der Dotterhautbildung infolge der $\mathrm{CO}_{2}$-Wirkung und nach längerem Verbleiben im Seewasser nicht durch dasselbe causale Moment bedingt ist. Naheliegend schien die Vermutung zu sein, daß, wenn die Eier längere Zeit im Seewasser liegen, vielleicht das Wasser mit der ron ibnen als Stoffiwechselprodukt ausgeschiedenen Kohlensäure teilweise gesättigt wird und die Eier dadurch die Fähigkeit der Membranerzeugung nilch der Befruchtung einbủßen.

konnte feststellen, daß die Eier, welche kiinstliche Dottermembranen gebildet haben, ahnliche Ziihigkeit des Protoplasmas zeigen. Es ist vielleicht anzanehmen. daß mit der Veränderung der physikalischen Eigenschaften des Protoplasmas auch der physiologische Zustand bzw. die Befruchtungsfihigkeit sich verändert.

1) Spezielles iber diese Veriinderungen kommt später zur sprache. 
Die positive Entscheidung dieser Vermutung bedarf jedoch weiterer experimenteller Priifung.

Die Hauptergebnisse der bisherigen Erwägungen uber den Ein$\mathrm{fluB}$ des mit $\mathrm{CO}_{2}$ gesättigten Seewassers anf die äußeren Befruchtungserscheinungen lassen sich folgendermaßen zusammenstellen:

1) Der Aufenthalt der Eier in mit $\mathrm{CO}_{2}$ gesättigtem Seewasser hat, wenn er bis zu 2 Minuten dauert, keinen wahrnehmbaren Einfluß auf die Befruchtungs- und Entwicklungsvorgänge.

2) Verbleiben die Eier 2--4 $\frac{1}{2}$ Minuten in demselben Seewasser und werden sie nachträglich in normales Seewasser ibertragen, so kann dadurch die Bildung einer Dotterhaut (J. LOEB) künstlich hervorgerufen werden. Solche Eier lassen sich nicht befruchten, wenn auch die Dotterhaut durch Schütteln entfernt worden ist.

3) Vor der Polyspermie wird das Ei nicht nur durch die Dotterhaut geschützt, sondern auch durch die inneren Veränderungen im $\mathbf{E i}$, welche sich gleichzeitig mit der Membranbildung abspielen.

4 Die Eier, welche 6-12 Minuten in dem mit $\mathrm{CO}_{2}$ gesättigten Seewasser rerblieben sind und nachher in gewöhnliches Seewasser übertragen wurden, lassen sich leicht befruchten, bilden aber keine abstehende Befruchtungsmembran.

5) Die Eier, welche die Eigenschaft der Dottermembranerzeugung: unter dem Einfluß von $\mathrm{CO}_{2}$ eingebüßt haben, verlieren trotzdem ihre Entwicklungsfähigkeit nicht und können eventuell nach gewissen Regulationsvorgängen normal gestaltete Embryonen bilden.

6) Das Einbüßen der Fähigkeit der Membranbildung ist kein die Polyspermie begünstigendes Moment.

3. Der Verlauf der Furchungsperiode, der Blastocoelbildung und der Kernsubstanzproduktion.

In den bisher beschriebenen Experimenten habe ich die EchinusEier mit dem vollkommen mit $\mathrm{CO}_{2}$ gesättigten Seewasser behandelt. Der Einwirkung dieser Flüssigkeit waren die Eier eine verhältnismäßig kurze Zeit ausgesetzt. In einer andern Reihe von Experimenten habe ich das mit $\mathrm{CO}_{2}$ gesättigte Seewasser mit dem normalen in folgenden Verhältnissen gemischt:

1) $5 \mathrm{ccm}$ des mit $\mathrm{CO}_{2}$ gesätt. Seew. $+45 \mathrm{ccm}$ des gewöhnl. Seewass.,

2) 10

3) 15 
4) $20 \mathrm{ccm}$ des mit $\mathrm{CO}_{2}$ gesätt. Seew. $+30 \mathrm{ccm}$ des gewöhnl. Seewass.,

5) 25

6) 30

7) 35

8) 40

9) 45

In diesen Lösungen ließ ich die Eier 6-7 Stunden liegen, nachher wurden die Eier in normales Seewasser übertragen. Es hat sich bei der Kontrolle einzelner Portionen herausgestellt, daß die Eier der 1. - 5. Serie ganz normal befruchtet wurden und alle die Dottermembran aufwiesen. In der 6. Serie hat nur ein Teil die Dottermembran gebildet, in der 7. und 8. Serie wurde von den Eiern die Befruchtungsmembran nie erzeugt.

Am nächsten Tage schwammen in den Kulturen $1-7$ die Blastulae. In der 8.-9. Kultur habe ich keine schwimmenden Blastulae gefunden ${ }^{1}$.

Im Verlauf der weiteren Entwicklung der Eier, welche 6-7 Stunden im $\mathrm{CO}_{2}$-haltigen Seewasser verbleiben, kann ein durchgreifender Unterschied im Vergleich mit dem normalen Entwicklungsverlauf schon bei der Beobachtung des lebenden Materials festgestellt werden. Wenn solche Eier eine vo n a ßen wahrnehmbare Furchung uiberhaupt durchmachen, werden in diesen Entwicklungsstadien nicht typische kugelige Blastomeren gebildet, sondern der Embryo besteht z. B. aus zwei halbkugeligen Zellen. Auch in späteren Furchungsstadien runden sich die Blastomeren nicht ab. Noch im 16-Zellenstadium behält der Embryo die primitive, vollkommen runde Gestalt der Eikugel. Trotzdem verläuft, wenn nur die Furchung äußerlich sichtbar war, die ganze weitere Entwicklung ganz normal.

Die Anzahl der Eier, welche die Furchung des Protoplasmas durchmachen, ist jedoch besonders bei stärkerer $\mathrm{CO}_{2}$-Konzentration (Kultur 7,8) sehr gering. Die Mehrzahl dieser Kulturen zeigt äußerlich überhaupt keine Spuren von Protoplasmateilung, so daß man bei fluchtiger Beobachtung den Eindruck gewinnt, daß sich das Ei nicht entwickelt. Unter stärkerer Vergrößerung kann man sich jedoch

1) Es muß hervorgehoben werden, daß auch in andern Kulturen ein 'Teil der Eier unentwickelt blieb, oder die Entwicklung gleich am Anfang stehen geblieben ist. Will man die Kultur weiter führen und es verhüten, daß die gesunden Embryonen durch die Zersetzungsprodukte der abgestorbenen vergiftet werden, so muß man die gesunden, schwimmenden Embryonen herausfischen und in frisches Seewasser ubertragen. 
schon am lebenden Material überzeugen, daß im Innern des Protoplasmas Kernteilung stattgefunden hat.

Die nähere Beschreibang solcher Eier scheint mir entbehrlich zu sein. DRIESCH (92) hat bei der veränderten Seewasserkonzentration und bei T'emperaturveränderungen, WILsON (01) bei der Ätherisation der Echinideneier die Erscheinung der Kernteilung ohne Zellteilung beschrieben und abgebildet.

Nach einigen Stunden erscheint die innere Partie dieser ungeteilten Kugel heller. Man kann daraus die Bildung des Blastocoels schon aus der Beobachtung am lebenden Material vermuten und bald darauf gewinnt man den Eindruck, daß sich Zellterritorien absondern. In etwas späteren Stadien, wenn das Blastocoel sich erweitert hat und die Wand desselben dünner und durchsichtiger geworden ist, sind oft im Innern des Blastocoels kleine Bläschen wahrnehmbar. Die Bedeutung dieser Gebilde läßt sich erst auf Grund der Schnittpräparate erklären (vgl. S. 314). Die Bläschen lassen sich noch in späteren Entwicklungsstadien zwischen dem Ecto- und Entoderm wahrnehmen. Ich habe solche winzige Kugeln im Gastrulaprisma, ja sogar im Pluteusstadium noch beobachtet.

Schon aus der Untersuchung des lebenden Materials geht hervor, daß unter dem Einfluß des $\mathrm{CO}_{2}$-haltigen Seewassers Störungen im Verlauf des Furchungsprozesses eingetreten sind, welche jedoch durch Regulationsvorg:̈nge beseitigt werden. Um sowohl die Veränderungen im Entwicklungsgang wie auch die Regulationsvorgänge näher kennen zu lernen, muß das fixierte Material an Schnitten untersucht werden.

Die ersten Serien der Eier, welche mit $\mathrm{CO}_{2}$-haltigem Seewasser behandelt waren, wurden vor der Befruchtung fixiert. Die Schnittpräparate beweisen, daß an den Eiern eine parthenogenetische Entwicklung beginnt. Im Protoplasma treten deutliche Strahlungen auf. Die Strahlungen sehen jedoch anders aus, als sie gewöhnlich bei dem Beginn der künstlichen Parthenogenese beschrieben werden. Die Strahlungen bei der künstlichen Parthenogenese der Seeigel (vgl. Morgax, 96, Wilsox, 01, Perrunkewitsch, 04) sind sehr zahlreich und im ganzen Protoplasma zerstreut. Hier treten nur zwei Strahlungen auf, wie bei der gewöhnlichen Mitose nach der Befruchtung. Dieser Umstand ist aus diesem Grunde von Wichtigkeit, da von manchen Autoren (Boveri, 02, S. 42) die Zahl der Strahlungen, welche am Anfang der Entwicklung zum Vorschein kommen, mit Recht als ein sehr wichtiger Unterschied zwischen der künstlichen Parthenogenese und der Befruchtung zitiert wurde. In der neuen Loesschen 
Methode ist also auch dieser Unterschied beseitigt und die Imitation der Befruchtung auch in dieser Hinsicht exakt.

Gleichzeitig treten auch die Veränderungen in der Kernstruktur auf. Hierbei muß ich bemerken, daß der parthenogenetischen Caryokinese keine KernvergröBerung vorangeht. Ich habe die Kernumrisse zahlreicher Eier, welche durch $\mathrm{CO}_{2}$ einen leichten Anstoß zur Parthenogenese bekommen haben, gemessen und mit dem Kernvolumen des unbefruchteten Eies verglichen, ohne jedoch eine Kernvergrößerung in dem mit $\mathrm{CO}_{2}$ behandelten Ei feststellen zu künnen.

Die Eier, bei welchen sich der Anstob zur Parthenogenese auf die oben beschriebene Weise geäußert hat, wurden besamt. Die Präparate der Befruchtungsstadien beweisen, daß Polyspermie trotz der Behandlung mit dem $\mathrm{CO}_{2}$-haltigen Seewasser nicht eingetreten ist. Die Eier sind hauptsächlich monosperm befruchtet. Im Verlauf des Befruchtungsprozesses lassen sich einige Abweichungen ron den normalen Vorgängen feststellen. In den Eiern, welche vorher durch längere Zeit mit $\mathrm{CO}_{2}$-haltigem Seewasser behandelt wurden, ist die Wanderung des Spermakopfes bedeutend verlangsamt. Oft trifft man nach beinahe zwei Stunden nach der Besamung den Spermakupf in einiger Entfernung vom Eikern liegen. Während dieser verlängerten Wanderung schwillt der Spermakopf an, so daß er vor der Copulation eine bläschenförmige Gestalt annimmt. Fig. 9 stellt das Bild des "Aufeinanderliegens * der Vorkerne dar. Wir sehen, daß beide Vorkerne bläschenförmige Gestalt und beinahe das gleiche Volumen zeigen. Bald darauf beginnt die Caryokinese.

In andern von mir beobachteten Fällen der Befruchtung der Eier, welche vorher mit $\mathrm{CO}_{2}$-haltigem Seewasser behandelt wurden, ist die Wanderung des Spermakerns noch mehr rerlangsamt, so daß es hier zur partiellen Befruchtung kommen kann. Ich habe auch Bilder gesehen, welche auf die Möglichkeit der selbständigen Spermakerncaryokinese obme vorherige Copulation der Vorkerne hinzuweisen scheinen.

Es ist nicht der Zweck dieser Arbeit, genau die cytologischen Vorgänge zu schildern, welche sich während der Befruchtung der zur Parthenogenese angeregten Eier in denselben abspielen. Für uns genügt die Feststellung der Tatsache, daß in der Mehrzahl der Fälle die Befruchtung normal oder mit geringen Abweichungen verlaufen kann.

Nach Beendigung des Befruchtungsvorganges beginnt die erste Caryokinese. Die Kernteilungsfiguren sind meist normal und bei einem kleinen Teil der Eier verlïnft auch die Zellleibsteilung ziemlich regel- 
mäßig. Jedoch bei dem weitaus größten Teil der Eier wird die Kernteilung von der Zellteilung nicht begleitet, so daß eine zweikernige Zelle resultiert. Die Kernteilung schreitet weiter ror. Im ungeteilten Plasmakörper bilden sich durch zweipolige mehrfache Mitosen mehrere Kerne aus Fig. 10. Hier und da sind im ungeteilten Plasmaterritorium auch mehrpolige Mitosen zu sehen.

Die Kernteilung obne Plasmateilung wurde sowohl bei der Bildung der Geschlechtselemente, wie auch bei den Entwicklungsprozessen mehrmals beobachtet. Von Flenuma $(80$, Auerbacil $(96)$ und ron mir:97) wurde die Hemmung der Zellleibsteilung bei der spermatogenese beschrieben und abgebildet, $O$. und R. Hentwig (87), Nonmaxx (96), Driesch (92), Wilsox (01), Lillie (02, 05), Scott (06), Treadwelu (07), Kostasecki (08) u. a. haben unter dem Linfuß rerschiedener : iußerer Faktoren die Kernteilung ohne Plasmateilung in den ersten Entwicklungsstadien der befruchteten oder zur künstlichen Parthenogenese angeregten Eier beschrieben. Die Kerne, welche durch die aufeinander folgenden Mitosen produziert werden, liegen hauptsächlich in der peripherischen Schicht des einheitlichen Plasmakörpers. Die Zahl der Kerne nimmt jedoch immer mehr zu und in späteren Stadien finden wir die Kerne auch in den centralen Teilen des Eiplasmas. Jetzt beginnt gewöhnlich die Verschmelzung der Kerne. Zwei, drei, oder noch mehrere Keme nähern sich einander und verscbmelzen zu einer morphologischen Einheit. Das Resultat der Verschmelzungsprozesse ist, daß in spïteren Furchungsstadien die Kerne, welche in einem einheitlichen Plasmaterritorium liegen, ron ganz verschiedener Größe sind (Fig. 11). Neben den einwertigen Kernen trifft man bedeutend größere, welche aus der Yerschmelzung ron z drei Kernen entstandeu sind. Ähnliche Verschmelzungsprozesse der Kerne wurden schon ron WILsox (01) bei den ätherisierten Toxopneustes-Eiern und neuerlich ron Kostanecki $(08)$ bei parthenogenetischer Hactra-Furchung beschrieben. Solche Kerne, welche durch Verschmelyung mehrerer einwertiger Kerne in den pflanzlichen Zellen entstanden sind, nennt Strasberger (07) Syncaryonten.

In den centralen Plasmateilen sind oft auch Riesenkerne zu sehen, deren Genese auf die Verschmelzung von mehreren Kernen zurickzuführen ist. Fig. 13 stellt den Verschmelzangsprozeß von mehreren dicht aufeinander liegenden Kernen dar. Oft können die gesamten in der centralen Eipartie gelegenen Kerne in einen centralen Riesenkern verschmelzen. In der peripherischen Plasmaschicht sind in solchem Fall die kleineren Eikerne gruppiert, in der Mitte liegen ein 
oder zwei große Kerne, um welche sich der bei weitem größte Plasmateil sammelt.

In meinen Präparaten habe ich oft auch Bilder gesehen, welche der Fig. 12 ähnlich sind. Wir sehen hier ein großes, einheitliches Plasmaterritorium, in welchem die Kerne von verschiedener Größe zu sehen sind. Ein verhältnismäßig kleiner Plasmateil, welcher einen Kern euthalten kann oder kernlos ist, hat sich von der gesamten ungeteilten Plasmamasse abreschnilrt. Diese Plasmapartie beteiligt sich nicht an der weiteren Entwicklung und wird in späteren Entwicklungsstadien ganz abgetrennt. Es ist nicbt ausgeschlossen, daß es eine Art Autotomie ist, welche bei den durch Schütteln verletzten Eiern von KRAHELSKa (0亏) beschrieben wurde.

Gleichzeitig mit dem Beginn der Kernverschmelzungspro/esse verläuft auch die simultane Teilung des bisher ungeteilten Protoplasmas. Gewöhnlich beginnt die Abgreuzung der Zellterritorien von der Peripherie des Eies durch das Anftreten ron Furchen, welche von der Oberfläche des Eies aus einschneiden. Oft sind infolgedessen einzelne Zellterritorien an der Peripherie schon abgegrenzt, fließen dagegen mit ihren inneren Partien in eine gemeinsame Plasmamasse zusammen. Bei der Beobachtung gewisser Furchungsstadien gewinnt man den Eindruck, als ob diese Eier nach dem superficiellen Furchungstypus sich teilten. Besonders auffällig ist jedoch dabei die Tatsache, daß die Größe des plasmatischen Territoriums, welches sich um die einzelnen Kerne sammelt, von der Größe des Kerns abhängig ist. Im Vorhergehenden habe ich darauf hingewiesen, daß die Kerne infolge der oft vorkommenden Verschmelzungsprozesse von ungleicher Größe sind. Aus den Figuren 13, 16, 17, 18, 19, 20 ist zu ersehen, daß bei der simultanen Plasmateilung das Massenverhältnis zwischen Kern und Protoplasma während der Furchung in gesetzmïßiger Weise geregelt wird.

Bekanntlich ist R. HerTwig (03) anf Grund eigner Beobachtungen an den Protozoen und auf Grund der Erwägung von verschiedenen Literaturangaben, besonders denjenigen von Boteri und Gerasinoff (01), zu der Überzeugung gekommen, daß man die komplizierten Wechselwirkungen zwischen Kern and Protoplasma nur durch die Annahme erklären kann, »daß jeder Zelle normalerweise eine bestimmte Correlation von Plasma und Kernmasse zukommt, welche wir kurz die ,Kernplasmarelation' nennen wollen $\kappa$.

Auf Grund seiner Untersuchungen an deu sich entwickelnden Echinideneiern hat sich Boreri $(05)$ den Anschaungen von R. HerTwig 
angeschlossen. Seine Zellenstudien an den dispermen und arrhenocaryotischen Echinidenembryonen bilden ebenfalls die Stütze für die Gesetze der Kernplasmarelation. BoverI ist auch der Ansicht: •Ein großer Kern vermag ceteris paribus einen größeren Zellleib zu versorgen als ein kleinerer. "Wenn die Chromatinquantität künstlich vergrößert oder verkleinert wird, so kommt es im Laufe der weiteren Entwicklung zu einer Regulation, welche einer richtigen Kernplasmarelation zustrebt: „Es kann die richtige Kernplasmarelation für verschiedene Chromatinmengen der Ausgangszellen einfach dadurch erreicht werden, daß sich im Fall von abnorm wenig Chromatin die Embryonalzellen öfter, im Fall einer abnorm großen Chromatinmenge weniger oft teilen als normalerweise « (Boveri).

Der Mechanismus der Regulation des Kernplasmaverhältnisses liegt in den von Boveri beschriebenen Fällen in der Normierung der aufeinander folgenden Zellteilungen. In unserm Fall verläuft die Regulation auf andre Weise: wenn die Kerne im ungeteilten Eiprotoplasma infolge der Verschmelzungsprozesse ungleiche Größe anfweisen und das Protoplasma sich simultan um diese Kerne verteilt, kann die Kernplasmarelation durch die Gruppierung verschiedener Plasmaquantitäten um die einzelnen Kerne hergestellt werden.

Dabei ist hervorzuheben, daß diese Art und Weise der Regulation nur den älteren Furchungsstadien zukommt. Wäre nämlich dieser Modus bei der simultanen Teilung auch in den frühesten Furehungsstadien möglich, so könnte man von vornherein postulieren, daß, wenn infolge der Polyspermie die simultane Eifurchung stattfindet, Blastomeren von ungleicher Größe resultieren. Es ist nämlich von Boveri (07) nachgewiesen worden, daß die nach der Polyspermie eintretenden mehrpoligen Mitosen eine ungleichmäßige Verteilung der Chromosomen veranlassen und infolgedessen ungleich große Blastomerenkerne resultieren.

Da jedoch trotzdem die plasmatischen Territorien, welche bei dieser simultanen Teilung entstehen, keinen Größenunterschied zeigen, muß daraus der Schluß gezogen werden, daß in diesen frühen Furchungsstadien die Herstellung der richtigen Kernplasmarelation durch entsprechende Verteilung von verschiedenen Plasmaquantitäten um die einzelnen Kerne nicht durchführbar ist.

Nachdem die Furchung vollendet ist, oder oft auch während die simultane Plasmateilung noch dauert, beginnt die Bildung des Blastocoels. Die Furchungshöhle bildet sich bei diesen Eiern meist nicht durch Auseinanderweichen von Zellelementen, da ja oft nur die 
peripher gelegenen Zellen vollständig abgegrenzt siud. Die Bildung des Blastocoels kommt hier vielmehr durch Kolliquation der inneren Plasmapartien zustande. Man sieht zunächst im Innern des Eies zahlreiche Vacuolen (Fig. 12); dieselben fließen dann in eine größere Höhle zusammen, welche mit durchsichtiger Substanz ausgefüllt ist.

Die Kolliquation des Protoplasmas schreitet weiter ror und nach einiger Zeit werden die ganzeu centralen Plasmapartien, welche einen »Riesenkern. (Fig. 14) oder die Riesenkerne (Fig. 15) ungeben, verflüssigt. Dieselben kommen dann in eine die Blastulahöble ausfüllende durchsichtige Flissigkeit zu liegen (Fig. 14, 15). Die Riesenkerne, welche der Verschmelzung mehrerer Kerne im Morulastadium ihre Entstehung rerdanken, können in Blastocoel der Degeneration anbeimfallen. Im Blastocoel der Fig. 14 sind Zerfallsprodukte eines solchen destruierten Kerns neben dem andern noch gesunden Srncaryon wahrnehmbar. In andern Fällen sind sie noch in späteren Entwicklungsstadien (im Gastrula- oder sogar im Prismastadium) zu seben. Diese Riesenkerne sahen am lebenden Material wie bläschenförmige Gebilde aus (vgl. S. 309).

Diese Art der Blastocoelbildung ist auch aus theoretiscben Riicksichten nicht ohne Bedeutung: Wird die Blastula als Endprodukt des Furchungsprozesses aufgefaßt, so geht ans der Betrachtung der Verlaufsarten der Gestaltungsrorgänge dieses Stadiums bei der Entwicklung der normalen und der mit $\mathrm{CO}_{2}$-haltigem Seewasser behandelten Eier hervor, daß das gleiche Endziel auf verschiedenen Wegen erreicht wird. Bei den normal sich entwickelnden Eiern beruht der morphogenetische Vorgang der Blastocoelbildung bekanntlich darauf, daß die Elemente auseinanderweichen und an der Bildung einer durchsichtigen Blastocoelflüssigkeit sich beteiligen. Dasselbe Gestaltungsendprodukt wird bei den Eiern, welche eine simultane Plasmateilung durchgemacht haben, durch eine Art der Rückbildung der centralen Plasmateile erreicht. In gewisser Hinsicht könnte dieser Prozeß als Regulation durch Destruktion aufgefaßt werden.

$\mathrm{Ob}$ es sich hier um eine echte Destruktion handelt, ist schwer mit vollkommener Sicherheit zu entscheiden, da es auf Grund unsrer technischen Untersuchungsmittel unmöglich ist zu entscheiden, was im Innern des Protoplasmas während der Umwandlung desselben in eine durchsichtige, die Furchungshöhle ausfüllende Flüssigkeit vorgeht. Immerhin verschwindet während dieser Vorgänge die sichtbare Struktur des Protoplasmas, so daß ein Teil desselben für die weiteren Gestaltungsrorg:inge als Bildungsmaterial in Wegfall kommt, welcher 
Vorgang im Sinne ron DrIEscir (01) eben »als Regulation durch Destruktion « bezeichnet werden kann.

Als Stütze für diese Dentung kann noch der Vergleich des Volumens der Blastulawand eines unter normalen Verhältnissen sich entwickelnden Echinidenkeimes mit dem Volumen der Blastula, welche aus dem mit $\mathrm{CO}_{2}$ behandelten Ei sich entwickelt hat, angeführt werden.

Das Volumen der Blastula, welche sich in normalen Verhältnissen entwickelt, beträgt 505620, das Volumen der Blastula, welche ans dem mit $\mathrm{CO}_{2}$-haltigem Seewasser behandelten Ei herstammt, beträgt 303600, also noch weniger als das Volumen des unbefruchteten Eies $(368770)$. Der Unterschied in der Größe dieser Blastulae beweist, daß ein Teil der Substanz bei dem regulatorischen Verlauf der Blastocoelbildung von dem Anteil an den Bildungsprozessen ausgeschlossen wurde. Es ist aus zahlreichen Literaturangaben längst bekaunt, daß die Entwicklung normal ablaufen kann, obschon ein Teil des Eies, bzw. ein Teil des schon in Entwicklung begriffenen Keimes, abgetrennt wird. Darauf basiert bekanntlich der Begriff der äquipotentiellen Systeme. In den bisherigen Untersuchungen wurde inmer ein Defekt an den peripherischen Teilen des Eies künstlich angesetzt. Die hier beschriebene Regulationsart, welche auf der Destruktion der centralen Partien des Embryos beruht, ist ein Beweis, daß auch nach solchen Defekten, welche die centralen Teile des Embryos von der weiteren Entwicklung ausschließen, die regulative Ausgestaltung der Larve möglich ist.

Bei der Beobachtung der beschriebenen Bilder erhebt sich die Frage, ob bei dieser Art der Kernvermehrung, welche von der Plasmateilung nicht begleitet wurde, dieselbe Quantität der Kernsubstanz produziert wird wie bei der normalen Furchung, bei welcher die Kern- und Plasmateilung gleichzeitig verlaufen. Die Resultate der Berechnungen, welche auf Grund der oben beschriebenen Methode durchgeführt wurden, ergaben, daß das Volumen der gesamten Kernsubstanzmasse in den normal sich entwickelnden Blastulen 30716 beträgt, in den Blastnlae dagegen, welche aus den Eiern herstammen, die mit dem $\mathrm{CO}_{2}$-haltigen Seewasser behandelt wurden, beträgt das Volumen der gesamten Kernsubstanzmasse 33224.

Ans dem Vergleich dieser Ziffern geht hervor, daß die Kernsubstanzproduktion bei der normalen Entwicklung und bei der zeitweise sistierten Plasmateilung beinahe mit derselben Intensität verläuft. Diese Tatsache scheint mir ans dem Grunde von Bedeutung zu sein, weil es theoretisch ron rornherein nicht ausgeschlossen wäre, 
daß in dem Furchungsprozeß ein Regulationsmoment für die Transformation des Protoplasmas in Kernsubstanz vorliegt. Aus den angeführten Ziffern ergibt sich jedoch, daß die Kernsubstanzproduktion von der Plasmateilung anabhängig ist.

Um die weitere Frage zu entscheiden, ob die embryonalen Organismen, die ihre Gestaltung den beschriebenen Regulationsprozessen verdanken, auch dieselbe Chromatinmasse enthalten wie die sich normal entwickelnden Blastulae, müßte man die Zahl der Kerne bzw. der durchgemachten Kernteilungen kennen und mit denen der normal sich entwickelnden Embryonen vergleichen. In Anbetracht der zahlreiehen Verschmelzungen der Kerne ist hier eine ganz genaue Bestimmung nicht durchfuhrbar. Jedoch ist es auf Grund von Präparaten (rgl. Fig. 14, 15) nach der approximativen Schätzung der Anzahl der Kerne und der Größe derselben doch ersichtlich, daß in diesen Embryonen zahlreiche Kernteilungen der zweiten Furchungsperiode (vgl. S. 288 und 289 stattgefunden haben. Wir wissen ans dem Vorhergehenden, daß durch die Teilungen dieser zweiten Periode die Kerusubstanz an Chromatingehalt bereichert wird, und so kann man indirekt vermutungsweise zu dem Schluß gelangen, daß die Hemmung der Plasmateilung während der Furchung keine besonderen Störungen in der Chromatinproduktion veranlaßt.

Man könute diesen Erörterungen vorwerfen, daß in den beschriebenen Fällen der Kernteilung ohne Zellteilung in den ersten Entwicklungsstadien doch eine Sonderung des Protoplasmas auch dann schon vorhanden ist, als sie morphologisch sich noch nicht wahruehmen läßt. Dagegen scheinen jedoch solche Fälle zu sprechen, in welchen das ganze Eiprotoplasma für die Ausbildung mitotischer Figuren verwendet wird. Einen solchen Fall hat in einer soeben erschienenen Arbeit Kostaxecki (08) beschrieben. In den unter der Einwirkung von hypertonischen Seewasserlösungen parthenogenetisch sich entwickelnden Eiern von Mactra hat Kostanecki Kernteilung ohne Zellteilung beobachtet. Im ungeteilten Ei liegen zahlreiche Kerne oder mehrpolige Mitosen, welche das ganze Ei eingenommen haben. In der Tatsache, daß für eine caryokinetische Figur das ganze plasmatische Territorium des Keimes aktiviert wurde, sehe ich den Beweis, daß das Protoplasma wirklich in einzelne Bezirke nicht gesondert wurde.

Bekanntlich wurde von R. Her rwig und T. Boveri das Problem der Kernplasmarelation auch für die Erklärung der die Zellteilung bewirkenden Momente verwertet. Aus den Untersuchungen iiber die 
s.Keime, die ihre Entwicklung mit mehr oder weniger als der normaleu Kernmenge oder mit abnormer Protoplasmamenge durchzuführen haben*, kommt Boveri (05) zu der Überzeugung, daß die Zahl der Teilungen keine für die einzelnen Zellenfolgen im voraus fixierte ist; » die Zellen teilen sich eben so lange, bis das richtige Verhältnis von Protoplasma und Kern so gut, wie es unter gegebenen Umständen möglich ist, erreicht ist.. Als die Zellteilung bewirkendes Moment ist also das Mißverhältnis zwischen Kern- und Protoplasmamenge, sowohl nach R. Hertwig wie nach T. Boveri, aufzufassen. Ich bin weit davon entfernt, die Wichtigkeit des Problems der Kernplasmarelation gering zu schätzen, und wir haben ja sogar in den Ergebnissen der Beobachtung der simultanen Teilung eine Stütze für die Behauptung gefunden, daß »jeder Zelle normalerweise eine gewisse Correlation von Plasma und Kernmasse zukommt " (R. HeRTwig), ich glaube jedoch kaum, daß man in dem Mißverbältnis zwischen Kern und Protoplasma in jeder einzelnen Zelle ein die Zellteilung bewirkendes Moment sehen könnte. Diese Behauptung basiert nämlich auf der Beobachtung, daß das rege Tempo der Zellteilung aufgehalten wird, wenn das richtige Verhältnis zwischen Kern und Protoplasma in den einzelnen Zellen (im Blastulastadium) erreicht wird. Im Vorhergehenden haben wir aber gesehen, daß die gauze Produktion der Kernsubstanz in einem einheitlichen Plasmaterritorium verlaufen kann und daß dadurch keine Störung in der Produktion der Kernsubstanz veranlaßt worden ist. Trotzdem, daß die Kernteilungen von Plasmateilungen nicht begleitet wurden, daB also infolgedessen die Zahl der Teilungen durch das Streben nach der Erreichung der Kernplasmarelation nicht normiert werden konnte, hat das rege Tempo nach Erreichung desselben Stadiums einen Stillstand erfahren. Diese Hypothese ist also zur Erklärung aller diesbezüglichen Erscheinungen sicher nicht ausreichend.

In innigem Zusammenhang mit diesem Problem steht die Frage, ob sich die bisherigen Anschaungen über die causalen Momente »der Beendigung der Furchung « mit den oben beschriebenen Befunden der Blastulabildung bei sistierter Plasmateilung vereinbaren lassen.

Mongan (95) war der erste, welcher bei Gelegenheit der Erforschung der Frage nach den kleinstmöglichen entwicklungsfähigen Eibruchstuicken das Problem der die Furchung beendigenden Faktoren berihht hat. Er hat zum erstenmal die wichtige Tatsache festgestellt, daß die Zellenzahl des abgefurchten Echinidenkeims konstant ist, und zwar hat er diese Zahl bei Echinus auf 1000 Zellen bestimmt. 
Auch hat Morgan zuerst die Vermutung ausgesprochen, daß »die Grenze der Teilbarkeit jeder Zelle durch das Verhältnis von Kern zu Protoplasma bestimmt ist . Er ist jedoch selbst auf Grund verschiedener Erwägungen davon abgekommen. DRIEsch (98) hat später darauf hingewiesen, daß diese Hypothese doch nicht so unhaltbar ist, wie es Morgan selbst glaubte.

Nachher hat Boveri (05) anf Grund seiner cytologischen Studien dieselbe Anschauung ausgesprochen 1 , er hält nämlich die Herstellung des richtigen Verhältnisses von Kern und Protoplasma für ein den Furchungsproze $\beta$ beendigendes Moment. In seiner Physiologie der Tierform, welche unlängst erschienen ist, schreibt DrIEsch: „Bei Seeigeln und Organismen ähnlicher Furchungsart ist der Grund für das Ende der Furchung in der Erreichung typischer, Kernplasmarelation' gegeben - der Prozeß wird also hier ans sich selbst sistiert. *

Es muß jedoch hervorgehoben werden, daß die Kernvermehrungsprozesse auch von selbst sistieren, wenn auch keine Plasmateilung die Kernteilung begleitet. Man kann also diese automatische Sistierung der Kernteilungen auf die Kernplasmarelation in einzelnen Zellen nicht zurïckführen. Aus der Tatsache, daß bei den normal abgefurteten Keimen und denjenigen Embryonen, bei welehen die Plasmateilung gehemmt war, doch am Ende der Furchung dieselbe absolute Kernsubstanzquantität gefunden wird, könnte man schließen, daß die Kernvermehrung von selbst sistiert, sobald nur eine bestimmte Kernsubstanzmenge durch Transformation des Protoplasmas entstanden ist, unabbängig davon, ob die Kernvermehrung im einheitlichen Plasmaterritorium stattgefunden hat, oder ob die Kernteilung von Plasmateilung begleitet war. Wir wissen jedoch aus den Bemerkungen des ersten Teils dieser Arbeit, daß fast die gesamte Kernsubstanzquantität schon im 64-Zellenstadium ausgebildet ist, wir haben weiter gesehen, daß die Zahl der Kerne, welche im ungeteilten Plasmaterritorinm entstehen, ungefähr dieselbe ist wie in den normalen Blastulen und die einwertigen Kerne ibrer Größe nach dem Volumen der Zellkerne der normalen Blastulae beinahe entsprechen. Daraus geht hervor, daß auch im ungeteilten Plasma die Kernteilungen der zweiten Furchungsperiode ablaufen - durch welche sich die Kernsubstanz an Chromatin bereichert. Erst wenn eine bestimmte Chromatinmenge durch die Transformation ausgebildet wird, kommt es zur Sistierung. 
der Kernvermehrung unabhängig davon, ob sich das Protoplasma in einzelne Zellen gesondert hat oder nicht.

Beziuglich der Beurteilung der die Furchung beendigenden Momente könnte man meiner Ansicht nach den Schluß ziehen, daß es sich dabei nicht um Erreichung einer bestimmten Kernplasmarelation in einzelnen Zellen handelt, sondern $d a B$ es die Ausproduzierung einer bestimmten Chromatinsubstanzmenge im ganzen Organis mus ist, welche die Sistierung des weiteren Kernvermehrungsprozesses veranlaßt.

Wenn wir jetzt weiter fragen, wie wird diese Quantität bestimmt, bei welcher ein relativer Stillstand in dem Furchungsverlauf eintritt, so scheint mir das Protoplasma es zu sein, welches in dieser Beziehung maßgebend ist. Die Chromatinsabstanzbildung kommt durch die Transformation des Protoplasmas zustande. Im Protoplasma sind die Materialsubstanzen dafür enthalten, welche von den successiv aufeinander folgenden Kerngenerationen verwendet werden. Von Bedentung ist in dieser Hinsicht noch die Tatsache, daß das Volumen des Protoplasmas am Ende der ersten Furchungsperiode (64 Zellen) rom Volumen des Eies nicht kleiner ist. Die Abnahme des Protoplasmas an Volumen beginnt eigentlich erst in der zweiten Furchungsperiode, und wenn wir am Ende der Furchung im Blastulastadium feststellen, daß die Quantität des Protoplasmas ungefähr um ein Drittel des ursprünglichen Volumens kleiner geworden ist, so ist es beinahe sicher, daß ein großer Teil dieses Protoplasmas zum Chromatin, welches eben in dieser Periode gebildet wird, sich transformiert hat. Die Furchung sistiert also von selbst, wenn ein bestimmter Teil des Protoplasmas sich zum Chromatin transformiert hat. Als endbestimmendes Moment halte ich also die Erreichnng einer bestimmten Relation der gesamten Plasmamenge zu der absoluten Chromatinsubstanzmasse im ganzen sich entwickelnden Keim.

Mir scheint es, daß der Nachdruck nicht auf die einzelnen Zellen zu legen ist, sondern der Keim als ein einheitliches Ganzes betrachtet werden soll. Es ist selbstrerständlich, daß, wenn die Zellteilung die Kernteilung begleitet — was bei der gewöhnlichen Furchung der Fall ist - die Erreichung einer bestimmten. Chromatinplasmarelation in jeder einzelnen Zelle und die Erreichung einer bestimmten Chromatinsubstanzmasse im ganzen embryonalen Organismus zusammenfallen. Wenn jedoch die Kernteilung ohne Zellteilung verläuft oder die Furchungsderivate ungleichmäßig sind, so 
wird sich der Unterschied in diesen zwei Auffassungen sofort ergeben.

DRIESCH hat meiner Ansicht nach vollkommen Recht, indem er sagt 1): "Alles in allem genommen, hat uns die neuere Forschung den Organismus in weit höherem Grade, als man es früher mochte, als ein Ganzes gezeigt; die Zellen sind oftmals Bausteine dieses Ganzen, aber mehr nicht, und oft nicht einmal das.*

\section{Die Mitosen der Riesenkerne (Syncaryonten2);.}

Wir haben gesehen, daß durch die Einwirkung von $\mathrm{CO}_{2}$-haltigem Seewasser die Zellleibsteilung gehemmt wird und infolgedessen zahlreiche Kerne im einheitlichen Plasmaterritorium liegen. Die beieinander liegenden Kerne können oft, wie oben hervorgehoben wurde, zu größeren Kernen verscbmelzen (vgl. auch WILson, 01, p. 370, und Kostanecki (08). Das Schicksal der Riesenkerne, welche in das Blastocoel hineingeraten, habe ich schon besprochen. Ein Teil der Kerne jedoch, welche ebenfalls der Verschmelzung mehrerer Kerne ihre Entstehung verdanken, bleibt im embryonalen Gewebe und kann an den weiteren Bildungsprozessen teilnehmen. Diese Kerne, welche sich mit einer großen, sich absondernden Plasmapartie umgeben, treten oft in Caryokinese ein. Der Verlauf der Mitose solcher Riesenkerne kann recht verschieden sein. Hier soll nur hervorgehoben werden, daß es entweder mehrpolige (Fig. 16) oder zweipolige Mitosen sind.

Auf Grund der Individualitätshypothese der Chromosomen wäre zu erwarten gewesen, daß die Zahl derselben von der Zahl der Kerne abhängt, welche an der Bildung der Syncaryonten teilgenommen haben. In der im vorigen Jahre veröffentlichten Arbeit hat Strasberger (07) nachgewiesen, daß durch Chloralhydrat (Methode von Nenec) »veranlaßte, also kuinstlich erzeugte, Syncaryonten an der ihnen zugewiesenen Chromosomenzahl festhalten «. In der Tat zeigen die Bilder der Caryokinesen der Riesenkerne of eine außerordentlich große Chromosomenzahl. Da die Zabl der Echinus-Chromosomen nach der Angabe von Boveri 36 beträgt, so ist leicht verständlich, daß in diesen Fällen, in welchen die drei- oder vierwertigen Syncaryonten in Caryokinese eintreten, die Zahl der Chromosomen sehr beträchtlich ist. Fig. 15 und 16 stellt eine solche mitotische Figur (Muttersternstadium) dar. Aus der Größe des plasmatischen Terri-

1) DRIESCH 07/ S. 73.

2: Dieser Name ist von Strasbcrger 07; eingeführt worden. 
toriums, in welchem die mitotische Figur liegt, ist sofort ersichtlich, daß dieses Territorium nicht durch den gewöhnlichen einwertigen Kern, sondern durch einen mehrwertigen versorgt sein muß.

Aus den Figuren 16 und 17 ist die beträchtliche Anzahl der Chromosomen auch ersichtlich, obschon nur ein Teil derselben auf der Durchschnittsebene lag und abgebildet wurde. Aber anßer diesen Typen der Caryokinese habe ich auch andre Mitosen der Syncaryonten gesehen, in welchen die Zahl der Chromosomen die normale Chromosomenanzahl der einwertigen Kerne nicht tuberschritt. Die einzelnen Chromosomen in der Mitose der Synearyonten waren jedoch entschieden größer als die Chromosomen in den Caryokinesen der einwertigen Kerne.

Auf der Fig. 18 ist das Bild eines Syncaryons aufgezeichnet, welcher in die Mitose eintritt; dasselbe Stadium sehen wir auch anf der Fig. 19. - Auf demselben Präparat (Fig. 19*) in der unmittelbaren Nachbarschaft ist auch der Anfang der Mitose eines einwertigen Kernes zu sehen. Die Differenz in der Größe dieser beiden Kerne und der Chromosomen derselben fällt sofort in die Augen. Die Übergangsformen zwischen diesen zwei Mitosentypen sind auch nicht selten (Fig. 20). Auf die theoretische Bedeutung dieses Typus der Mitose, welcher mit der Hypothese der Individualität der Chromosomen sich nicht ohne weiteres vereinbaren läßt, möchte ich hier nicht näher eingehen, da ich es an andern Orten zu tun beabsichtige. Ich möchte hier nur auf die Anschauung von STRASBURGer (07, S. 502) hinweisen: "Wo die Zahl der Chromosomen stetig oder nur gelegentlich infolge unterbliebener Trennung gegebener Einheiten eine Änderung erfährt, spricht das nicht gegen die Individualität der Chromosomen.* Anderseits werden solche Fälle auch anders gedeutet. FICK (07) schreibt in seiner im vorigen Jahre veröffentlichten Arbeit, in welcher er die Literatur der Chromosomenindividualitätshypothese kritisch bespricht: "Alle diese Fälle beweisen, falls die Deutnng der Entstehung zutrifft, nur die Aufrechterhaltung der Chromosomenzahl selbst unter abnormen Umständen, keineswegs aber die Erhaltung individueller Chromosomen ".

Das Resultat der Mitosen ist nicht nur die Vermehrung der Kerne, sondern auch in den Fällen, in welchen die mehrpoligen Mitosen (Fig. 16) sich bilden, die Verteilung der Kernsubstanz, welche in einem Riesenkern gesammelt war, auf mehrere kleinere Kerne. Da gleichzeitig auch das große Plasmaterritorium sich teilen kann, ist die Regulation der inneren struktuellen Verhältnisse möglich. Sehr oft 
kommen aber während der Mitosen der Riesenkerne die Destruktionsanzeichen vor, und nachher zerfallen die embryonalen Organismen and sterben ab.

\section{Andre Regulationstypen, weitere Entwicklung.}

Der Regulationsvorgang, den wir im bisherigen geschildert haben, führt zur Ausbildung einer sonst normalen, nur verkleinerten Blastula. Wir haben gesehen, daß die Kolliquation der centralen Teile die Ausbildung des Blastocoels ergeben hat. In andern Fällen sieht man, daß nach der simultanen Plasmateilung eine gewisse Zellenzahl im Innern und auf der Peripherie der Morula der Kolliquation anheimfällt, so daß ein länglicher, an der Peripherie der Morula sich verbreiternder Spalt gebildet wird (vgl. Fig. 21). Im Innern dieses Spaltes sind noch die Degenerationsprodukte hier und da wahrnehmbar (Fig. 21). Durch diesen Spalt wird der ganze Keim in zwei Hälften geteilt, welche nur durch eine schmale Zellenleiste miteinander zusammenhängen.

In den beiden Hälften des Keimes entwickeln sich jetzt durch Auseinanderweichen der Zellen oder durch weitere Destruktion der Zellelemente zwei kleine Blastocoele. Ein solches Bild stellt Fig. 22 dar. Eine gewisse Ähnlichkeit mit dem Bilde einer Invaginationsgastrula läßt sich aus dieser Figur sofort ersehen. Daß es aber in der Tat keine Gastrula ist, davon überzeugt die Beobachtung am lebenden Material und die Durchmusterung der Präparatenserien. Die Präparate beweisen, daß in späteren Entwicklungsstadien sich die Zellen in beiden Keimpartien derart ordnen, daß aus einem einheitlichen Keim zwei kleine Blastulae entstehen. Die Beobachtung in vivo ergibt, daß in solchen Kulturen die kleinen Zwillingsblastulae massenhaft herumschwimmen.

Aber ein solches Gebilde, wie es Fig. 22 veranschaulicht, rermag sich noch zu einer einheitlichen Blastula zu regulieren. Fig. 23 stellt ein solches Regulationsbild dar. Wir sehen, daß hier die »scheinbare Einstülpung « der äußeren Wand bedeutend seichter geworden ist, und daß die Zellen, welche ihre Wand bildeten, im Blastocoel auseinandergehen und dort wahrscheinlich degenerieren.

Wir haben also gesehen, daß nach den Störungen, welche durch Einwirkang von $\mathrm{CO}_{2}$ im furchenden Echinidenkeim eingetreten sind, die Regulationserscheinungen auf verschiedene Weise verlaufen sind, und immer dabei die normal gestaltete Blastula (wenn auch bedeutend kleiner) resultiert. Unabhängig daron, wie die Blastula entstanden 
ist, verläuft die weitere Entwicklung meist nach dem gewöhnlichen Typus. Es muß nur hervorgehoben werden, daß viele relativ regulierte und morphologisch normal aussehende Keime sich entwicklungsunfähig erwiesen haben. An solchen erkrankten Keimen konnte man im Blastocoel unregelmäßige Anhäufungen von dunkel anssehenden Körnern und Ballen wahrnehmen, woranf dann gewöhnlich die betreffenden Embryonen abgestorben sind. Diejenigen Keime, welche glïcklich das Gastrulastadium erreichten, entwickeln sich gewöhnlich auch weiter bis zum Pluteusstadium.

\section{Zusammenfassung der letzten Kapitel ${ }^{1}$.}

Die Ergebnisse der Lntersuchung der Furchungsperiode, der Blastocoelbildung und der Kernsubstanzproduktion in den Embryonen, welche sich aus den mit $\mathrm{CO}_{2}$-baltigem Seewasser behandelten Eiern entwickelt haben, lassen sich folgendermaßen zusammenstellen:

1) Nach einer entsprechend langen Exponierung der Eier tritt nach der Befruchtung die Kernteilung ohne Zellteilung auf.

2) Im Laufe dieses Prozesses findet oft die Bildung der Syncaryonten im einheitlichen Plasmaterritorium statt, welche der Verschmelzung oft mehrerer einwertigen Kerne ihre Entstehung verdanken.

3) Die Syncaryonten können durch mehrpolige oder bipolare Mitosen sich weiter teilen. Im ersten Fall kann eine regulative Verteilung der in den Riesenkernen enthaltenen Kernsubstanz auf mehrere kleinere Kerne zustande kommen. Die central gelegenen Riesenkerne geraten oft in das sich bildende Blastocoel.

4) Nachdem mehrere Kerne im einheitlichen Plasmaterritorium sich gebildet haben, beginnt die simultane Plasmateilung. Dabei läßt sich feststellen, daß die Größe der plasmatischen Territorien, welche sich um die einzelnen Kerne sammeln, von der Größe der betreffenden Kerne abhängig ist. Diese Tatsache steht mit den Gesetzen der quantitativen Kernplasmarelation im Einklang.

5) Das Blastocoel kann nur selten durch Auseinanderweichen der Zellen entstehen, gewöhnlich wird es durch die Destruktion der centralen Partien des Keimes gebildet.

6) Eine derartige Ausschließung der centralen Partien des Eies

1 Am Ende jedes Kapitels des ersten Teils und am Schluß des ersten Kapitels des zweiten Teils ist schon die Zusammenstellung der Resultate gegeben. 
von der Teilnahme an der Entwicklung beeinträchtigt die normale Gestaltung der rerkleinerten Larve nicht.

7) Die Syncaryonten können, wenn sie in das Blastocoel geraten, der Degeneration anheimfallen, sonst können sie sich durch bipolare oder mehrpolige Mitosen weiter teilen. Die mehrpoligen Mitosen können eine regulative Verteilung der Kernsubstanz auf kleinere Kerne zur Folge haben.

8) Die Zahl der Chromosomen bei den Mitosen von Syncaryonten kann eventuell die Zahl der Chromosomen der einwertigen Kerne nicht iberschreiten. Die Chromosomen sind aber in diesem Fall bedeutend größer als die Chromosomen der einwertigen Kerne.

9) Als endbestimmendes Moment bei dem Furchungsprozeß ist die Erreichung einer bestimmten Relation der gesamten Plasmamenge zu der absoluten Chromatinsubstanzmasse im ganzen sich entwickelnden Keim zu betrachten.

\section{Literaturverzeichnis.}

Acerbach. 1896. Untersuchungen uber die Spermatogenese von Palludina viripara. Jen. Zeitschr. f. Naturn. Bd. 30.

Boveri. T. 1902. Das Problem der Befiuchtung. Jena.

- 1905. Zellenstudien. V. Jena. 1907. Zellenstudien. VI. Jena.

DeLAGE, Y. 1902. L'acide carbonique comme agent de choix de la parthénogénèse expérimentale. Compt. rend. Acad. des Sc. Paris. T. 135.

__ 1902. Nouvelles recherches sur la parthénogénèse expérimentale chez Asterias glacialis. Arch. de Zoolog. expérimentale. T. 10.

Driesch, H. 1892. Entwicklungsmechanische Studien. IV, Zeitschr. f. wiss. Zool. Bd. 55 .

1893. Entwicklungsmechanische Studien. VIII. Mitt. d. Zool. Station zu Neapel. Bd. 11.

- 1898. Yon ded Beendigung morphogener Elementarprozesse. Dieses Arch. Bd. 6.

-_ 1900. Die isolierten Blastomeren des Echinidenkeimes. Dieses Arch. Bd. 10.

- - 1901. Die organischen Regulationen. Leipzig.

- 1906. Die Physiologie der tierischen Form. Ergebn. d. Physiologie. Bd. 5.

FICE, R. 1907. Vererbungsfragen. Reductions- und Chromosomenhypothesen, Bastardregeln. Ergebn. d. Anat. n. Entwicklungsgesch. Bd. 16.

Fuemming, W. 1880. Beiträge zur Kenntnis der Zelle und ihrer Lebenserscheinungen. Arch. f. mikr. Anat. Bd. 17.

Gerassmow, J. 1901. Die Abhängigkeit del Größe der Zelle von der Menge ihrer Kernmasse. Zeitschr. f. allgem. Physiologie. Bd. 1.

GodLEWski, E., jun. 1897. Über mehrfache bipolare Mitose bei der Spermatogenese von Helix pomatia. Anz. d. Akad. d. Wissensch. in Krakau. 1897. 1906. Intersuchungen über die Bastardierung der Echiniden- und Crinoidenfamilie. Dieses Arch. Bd. 20. 
HeRBST, C. 1898. Über zwei Fehlerquellen beim Nachweis der Unentbehrlichkeit von Phosphor und Eisen für die Entwicklung der Seeigellarven. Dieses Arch. Bd. 7.

- 1904. Über die zur Entwicklung der Seeigellarven notwendigen anorganischen Stoffe, ihre Rolle und ihre Vertretbarkeit. Ebenda. Bd. 17.

_- 1906. Vererbungsstudien. IV. Ebenda. Bd. 22.

Hertwic, O. und R. 1887. Über den Befruchtungs- und Teilungsvorgang des tierischen Eies unter dem Einfluß äußerer Agentien. Jen. Zeitschr. f. Naturw. Bd. 20.

Hertwig, R. 1903. Über das Wechselverhältnis von Kern und Protoplasma. München, Lehmann.

- 1903. Über Correlation von Zell- und Kerngröße und ihre Bedeutung für die geschlechtliche Differenzierung und die Teilung der Zelle. Biolog. Centralbl. Bd. 23.

KostaneCKI, K. 1908. Mitotische Kernteilung ohne Zellteilung in künstlich parthenogenetisch sich entwickelnden Eiern von Mactra. Bull. de l'Acad. des Sc. de Cracovie.

- 1908. Zur Morphologie der künstlichen parthenogenetischen Entwicklung bei Mactra. Arch. f. mikr. Anat. Bd. 76.

Krahelska, M. 1905̆. Zaplodnienie odłamków jaj jeżoweów i pierwsze okresy ich rozwoju. Rozpr. Akad. Um. Wydz. mat. przyr. Tom. 45.

Dasselbe französisch: Sur le développement mérogonique des œufs du Psammechinus. Bullet. de l'Acad. des Se. de Cracovie.

Liclie, F. R. 1902. Differentiation without Cleavage in the Egg of the Annelid Chaetopterus pergamentacens. Dieses Arch. Bd. 14.

- 1905. Observations and Experiments concerning the elementary phenomena of embryonic development in Chaetopterus. Journ. of Experiment. Zoology. Vol. 3.

LoEb, J. 1895. Über Kernteilung ohne Zellteilung. Dieses Arch. Bd. 2.

- 1898. Über den Einfluß vou Alkalien und Säuren auf die embryonale Entwicklung und das Wachstum. Ebenda. Bd. 7.

- 1905. On an improved Nethod of artificial Parthenogenesis. I, II, III Communications.; University of California Publications. Physiology. Vol. 2. 1905. Artificial Membrane Formation and Mechanical Fertilisation in a Starfish (Asterina). Ebenda.

1906. On the Necessity of the Presence of free Oxygen in the hypertonic Sea-water for the Production of artificial Parthenogenesis. Ebenda.

- 1906. Die Dynamik der Lebenserscheinungen. Leipzig, Barth.

—. 1906. Untersuchungen über künstliche Parthenogenese. Leipzig, Barth. Herausgegeben von E. SchwalBe.

-__ 1907. Weitere Versuche über die Notwendigkeit von freiem Sauerstoff für die entwicklungserregende Wirkung hypertonischer Lösungen. PfLüGers Arch. Bd. 118.

MarCUS, H. 1906. Über die Wirkung der Temperatur auf die Furchung bei Seeigeleiern. Dieses Arch. Bd. 22.

Morgan, T. H. 1895. Studies of the sartials Larvae of Sphaerechinns. Dieses Arch. Bd. 2.

- 1896. The Production of Artificial Astrosphaeres. Dieses Arch. Bd. 3.

Pexer, K. 1905. Untersuehungen iiber individuelle Variationen in der tierischen Entwicklung. Sitzungsber. d. Akad. ju Berlin. 
Petrunkewitsch, A. 1904. Künstliche Parthenogenese. Zool. Jahrb. Suppl. VII. Festschr. für Weismiann.

Sснміdт, H. 1904. Zur Kenntnis der Larvenentwicklung von Echinus microtuberculatus. Verh. der phys.-med. Ges. Würzburg. N. F. Bd. 36.

ScoTT, J. W. 1905. Morphology of the parthenogenetic development of Amphitrite. Journ. of Experiment. Zoology. Vol. 3.

Strasburger, E. 1907. Über die Individualität der Chromosomen und die Pfropfhybridenfrage. Jahrb. f. wiss. Botanik. Bd. 44.

Wilson, E. 1901. Experimental Studies in Cytology. II and III. Dieses Arch. Bd. 13.

\section{Erklärung der Abbildungen.}

Tafel III und IV.

Die Abbildungen sind von Herrn Stanislaw Weigner vermittels des Abseschen Zeichenapparates entworfen. Fig. 1-8 ist unter 1000 facher Vergrößerung, die übrigen etwa 700.

Fig. 1. Die Kerne der unbefruchteten Echinus-Eier.

Fig. 2. Die Kerne des Zweizellenstadiums.

Fig. 3. Die Kerne des Vierzellenstadiums.

Fig. 4. Die Kerne des 32-Blastomerenstadiums.

Fig. 5. Die Kerne des 64-Blastomerenstadiums.

Fig. 6. Die Kerne im Blastulastadium.

Fig. 7. Die Kerne im Pluteusstadium.

Fig. $8 a$. Die Kerne der Plutei-»Kältekulturs.

Fig. $8 b$. Die Kerne der Plutei-s Wirmekultur*.

Fig. 9. Die Befruchtung des mit $\mathrm{CO}_{2}$-haltigem Seewasser behandelten Eies. Der Spermakopf ist bläschenförmig geworden, da er während der verlängerten

Wanderang durch das Eiprotoplasma stark angeschwollen ist.

Fig. 10. Die Kernteilung ohne Zellleibsteilung.

Fig. 11. Mehrere Kerne im ungeteilten Zellleib.

Fig. 12. Mehrere Kerne im ungeteilten Zellleib, ein kleiner Plasmateil mit einem Kern wird abgetrennt.

Fig. 13. Der Verschmelzungsproze $B$ der central gelegenen Kerne. Beginn der Blastocoelbildung.

Fig. 14. Blastula, in welcher das Blastocoel durch Destruktion innerer Partien entstanden ist. Im Blastocoel ist ein Riesenkern (Syncaryon) zu sehen.

Fig. 15. Blastula wie Fig. 13. Im Blastocoel sind zwei Syncaryonten wahrnehmbar.

Fig. 16. Dreipolige Mitose eines Syncaryons, zu welchem ein großes Plasmaterritorium gehürt.

Fig. 17. Zweipolige Mitose eines Syncaryons. Die Chromosomen sind klein, ihre Anzahl sehr betrïchtlich.

Fig. 18. Anfangsstadium der Mitose eines Syncaryons. Die Zahl der Chromosomen ist geringer, die Chromosomen größer.

Fig. 19. Ähnliches Stadium wie Fig. 17, die Mitose des einwertigen Kernes.

Fig. 20. Die Mitose eines Syncaryons.

Fig. 21 und 22. Die Bildung der Zwillingsblastulae.

Fig. 23. Regulation zu einer einheitlichen Blastula. 
है

$$
\begin{aligned}
& .000 \\
& 000 \\
& 00
\end{aligned}
$$

$\dot{\infty}$

$$
\begin{array}{ccccc}
0 & 0 & 0 & 0 & 0 \\
& 0 & 0 & \\
0 & 0 & 0 & 0 & 0 \\
0 & & 0 & 0 & 0 \\
0 & 0 & 0 & 0 & 0
\end{array}
$$

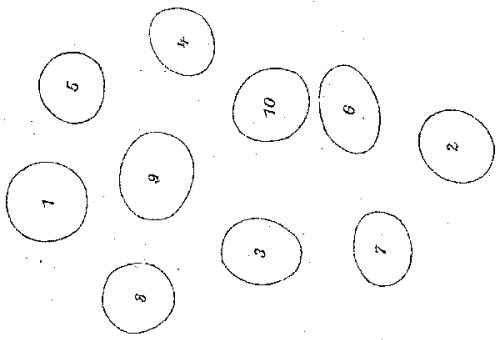

0.000

$0^{\circ} 0^{\circ} 0$

$\infty 00000$

$$
\begin{array}{cccc}
0 & 0 & 0 & \\
0 & 0 & 0 & 0 \\
& 0 & 0 & 0 \\
0 & 0 & 0 & 0 \\
\therefore & 0 & 0 & 0
\end{array}
$$

$(\infty) \infty \infty$ $(0, \infty)$

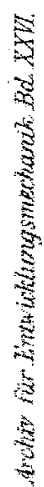

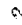

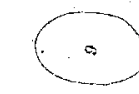

$$
\operatorname{coc}_{000} 0
$$

$(\infty)$

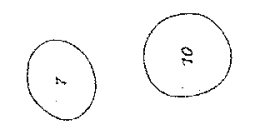

(a) ( )

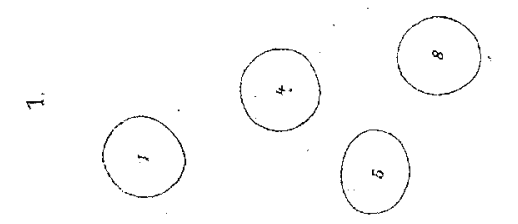

$\circ \quad 0000$

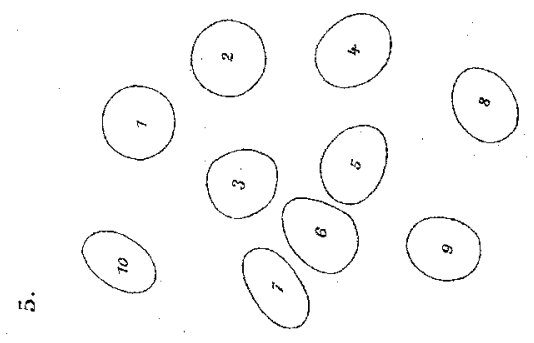




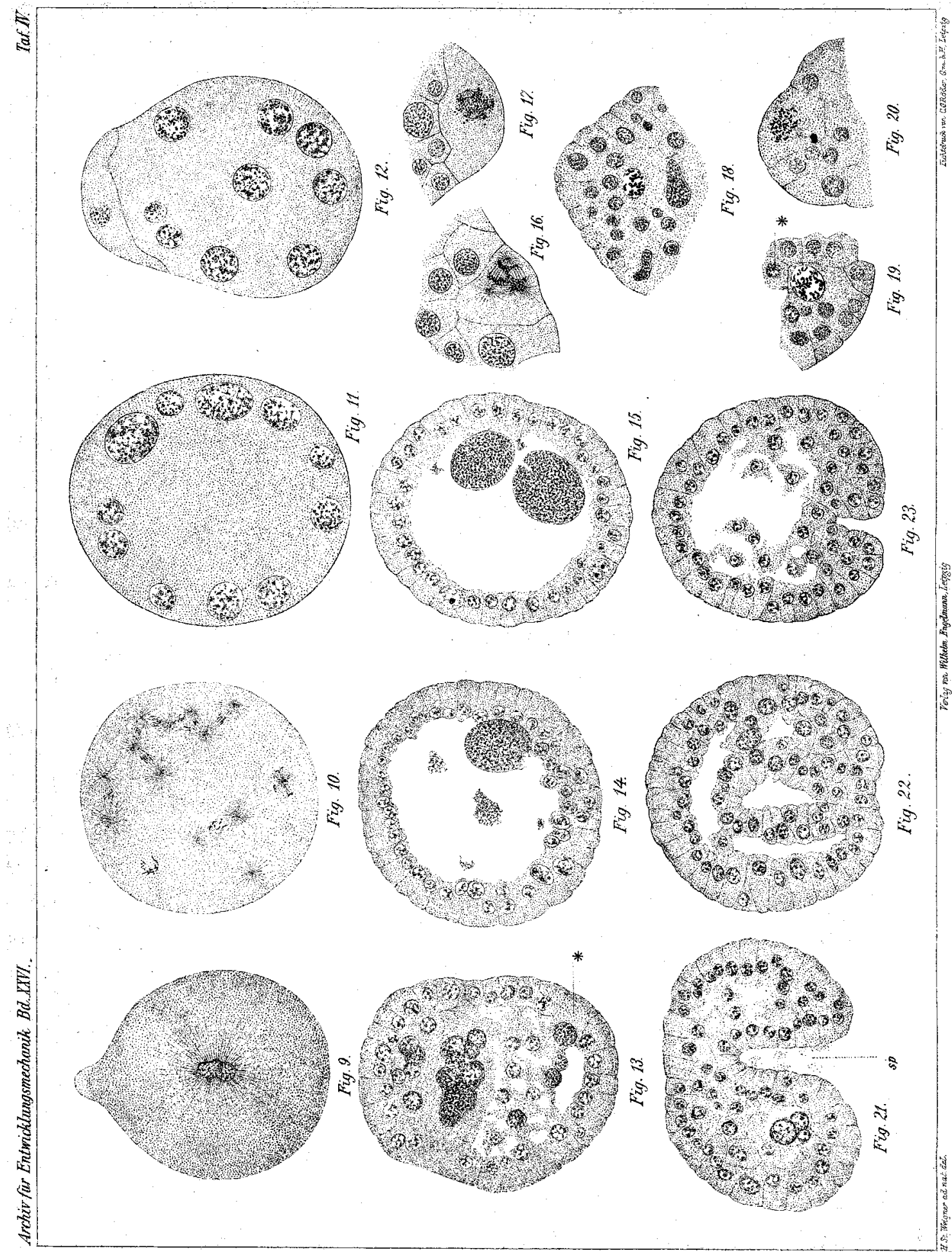




\section{Nachtrag.}

Als das Manuskript dieser Arbeit schon druckfertig war, habe ich dank der Guite des Herrn Geheimrat Prof. Dr. R. Hertwig den Separatabdruck seiner soeben erschienenen, für mich äußerst wichtigen Arbeit: » ̈̈ ber neue Probleme der Zellenlehre« 1 ) 1908 bekommen. Ich wollte den Text des Manuskriptes nicht mehr ändern, halte es aber doch für meine Pflicht, auf einige meiner vorliegenden Publikation nahestehende Punkte hier noch einzugehen.

1) Hertwig ist in seiner Arbeit auf Grund der von R. ERDMaxx auf seine Veranlassung ausgeführten Messungen der Chromosomen in einzelnen Entwicklungsstadien von Strongylocentrotus lividus zum Schluß gekonımen, daß im Laufe der Entwicklung die Chromosomengröße »eine ganz bedentende Abnahme erfährt". Ich habe in meinen Deduktionen tiber die Bedeutung der zweiten Furchungsperiode mich auf die von Boveri aufgestellte und bisher allgemein anerkannte Lchre gestuitzt, daß die Chromosomengröße während der ganzen Entwicklung im wesentlichen die gleiche bleibt. Vorausgesetzt also, daß die Größe der Chromosomen sich nicht verändert, habe ich aus meinen Untersuchungsergebnissen den Schluß gezogen, daß die Kernvermehrung der zweiten Furchungsperiode die Bereicherung der früher ausgebildeten Chromatinsubstanz zur Folge hat. Nun kann sich die Frage erheben, ob in Anbetracht der von R. Hertwig aufgestellten Regel, daß die Chromosomen in Laufe der Entwicklung sich verkleinern, meine oben angegebene Anschauung sich aufrecht erhalten läßt. Um sie auf ihre Richtigkeit zu prüfen, versuchen wir an der Hand der von R. Erumaxs gelieferten Ziffern die Berechnung der absoluten Quantität der Chromatinsubstanz am Ende der ersten Furchungsperiode (64 Zellen) und im Blastulastadium durchzuführen:

Die Größe eines Chromosoms in Zimmertemperatur im 64-Zellenstadium beträgt 3,46. Da in einem Kern 36 Chromosomen - nach Boveri - enthalten sind, so beträgt die absolute Chromatinquantität eines Kernes 123,56 - die absolute Chromatinquantität des ganzen Keimes beträgt also im 64-Zellenstadium $123,56 \times 64=7907,84$.

Aus meinen Messungen hat sich herausgestellt, daß die Kernsubstanz im Blastulastadium ungefähr in derselben Quantität wie im 64-Zellenstadium enthalten, aber auf größere Kernenanzahl verteilt ist. Wir haben z. B. in Zimmertemperatur 1256 Kerne gefunden.

1) Archir fiir Zellforschung. Bd. I. 1908. 
328 Emil Godlewski jun., Plasma u. Kernsubstanz in d. Entwickl. der Echiniden.

Die Größe eines Chromosoms beträgt nach den Angaben von HerTwig (ans der Arbeit von ERdMans) im Blastulastadium 2,41, also in den gesamten 1256 Kernen beträgt die absolute Chromatinsubstanzquantität $2,41 \times 36 \times 125,6=101434,56$. Wir sehen also, daß dieselbe Kernsubstanzquantität, welche im 64-Zellenstadium 7907 Chromatinmasse enthielt, im Blastulastadium bedeutend reicher an Chromatin geworden ist: wir finden nämlich 101434 Chromatinmasse.

2) Auf Grund der Voraussetzung, daß die Größe der Chromosomen von den änßeren Einflitssen unabhängig ist, und auf Grund der anf S. 315 und 316 angegebenen Erörterungen, bin ich zu dem Schluß gekommen, daß die Produktion der absoluten Chromatinquantität von äußeren Bedingungen beeinflußt wird. HeRTwig gibt jedoch an, daß die Größe der Chromosomen durch die Temperatur beeinflußt werden kann. Es ist also nicht ausgeschlossen, daß die Veränderungen in den Chromosomengrößen die Veränderungen in der Kernenanzahl kompensieren werden - in diesem Falle wäre also der Chromatingehalt konstant und von der Temperatur unabhängig. Etwas positives läßt sich aber vorläufig nicht aussagen.

3) Auf Grund der Beobachtung der Kernteilung ohne Zellteilung: bin ich zu der Anschauung gekommen, daß die Beendigung der Furchungsprozesse nicht durch das Erreichen der Kernplasmarelation veranlaßt ist, sondern daß der Furchungsprozeß von selbst sistiert, wenn ein gewisser Teil der gesamten Plasmamasse sich zum Chromatin transformiert hat. In der Anmerkung S. 7 gibt HERTwiG an: »daß möglicherweise nicht die Kernplasmarelation als solche die morphogenetischen Prozesse auslöst, sondern vielleicht die Beschaffenheit des Zellplasmas, welche durch die beständige Abgabe von Substanzen an die Kerne notgedrungen während des Furchungsprozesses eine Veränderung seiner Beschaffenheit erfahren muß . Meine auf anderm Wege unabhängig gewonnenen Resultate bilden also eine gewisse Analogie und Bestätigung der neuen Hertwigschen Anschaung. 